\title{
A CUTTING PLANE ALGORITHM FOR A CLUSTERING PROBLEM
}

\author{
M. GRÖTSCHEL
}

Institut für Mathematik, Universität Augsburg, Memminger Str. 6, 8900 Augsburg, West Germany

\section{Y. WAKABAYASHI}

Universidade de São Paulo, Instituto de Mathemática e Estatística, Caixa Postal 20.570

(Agência Iguatemi), 01498 São Paulo SP, Brazil

Received 12 November 1987

Revised manuscript received 12 April 1988

In this paper we consider a clustering problem that arises in qualitative data analysis. This problem can be transformed to a combinatorial optimization problem, the clique partitioning problem. We have studied the latter problem from a polyhedral point of view and determined large classes of facets of the associated polytope. These theoretical results are utilized in this paper. We describe a cutting plane algorithm that is based on the simplex method and uses exact and heuristic separation routines for some of the classes of facets mentioned before. We discuss some details of the implementation of our code and present our computational results. We mention applications from, e.g., zoology, economics, and the political sciences.

\section{Introduction}

The need of analysing data that arise from the measurement of a number of characteristics (or attributes) associated with each object of a given set, occurs very frequently in sociology, zoology, economics, and many other sciences. The areas of study concerned with this type of problem are known as data analysis, multivariate analysis, and taxonomy.

We consider here a problem occurring in qualitative data analysis, of the following type.

Given a data set consisting of the description of a set of objects with respect to a number of characteristics, find a best partition of the object set into "homogeneous" disjoint classes (or clusters).

In this paper we give a precise formulation of this clustering problem and show how it can be reduced to a graph optimization problem which we call clique partitioning problem (CPP). This is done in Section 2. In Section 3 we summarize some results of Grötschel and Wakabayashi (1987) on the polyhedron associated with the CPP. In Section 4 we describe a cutting plane algorithm for CPP which is based on these theoretical results. Finally, in Section 5 we report on the computational results with our code. Many applications from zoology, marketing, and the 
political sciences are given and the optimization process for each of these applications is illustrated.

\section{Definitions and notation}

We assume that the reader is familiar with the basic concepts of graph theory. The definitions not given here can be found in Bondy and Murty (1976). All graphs we consider are simple. We denote a graph $G$ with node set $V$ and edge set $E$ by $G=(V, E)$. An edge $e$ with endnodes $u$ and $v$ is denoted by $u v$. If $S$ is a node set of $G=(V, E)$ then we denote the set of edges in $G$ with both endnodes in $S$ by $E(S)$, that is,

$$
E(S)=\{u v \in E \mid u, v \in S\} .
$$

Moreover, if $S_{1}, \ldots, S_{k}$ are subsets of $V$ then

$$
E\left(S_{1}, \ldots, S_{k}\right):=\bigcup_{i=1}^{k} E\left(S_{i}\right)
$$

If $S, T \subseteq V$ and $S \cap T=\emptyset$ then

$$
[S: T]:=\{u v \mid u \in S, v \in T\}
$$

denotes the set of edges with one endnode in $S$ and the other in $T$.

A graph is called complete if every pair of its nodes is linked by an edge. A clique is a subgraph of a graph that is complete (a clique is not necessarily a maximal complete subgraph). We will denote the (up to isomorphism unique) complete graph with $n$ nodes by $K_{n}=\left(V_{n}, E_{n}\right)$.

We say that $\Gamma=\left\{W_{1}, \ldots, W_{k}\right\}$ is a partition of $V$ if $W_{i} \cap W_{j}=\emptyset$ for $1 \leqslant i<j \leqslant k$, $V=W_{1} \cup \cdots \cup W_{k}$ and $W_{i} \neq \emptyset$ for all $i$.

A set $A$ of edges in a graph $G=(V, E)$ is called a clique partitioning of $G$ if there is a partition $\Gamma=\left\{W_{1}, \ldots W_{k}\right\}$ of $V$ such that $A=E\left(W_{1}, \ldots, W_{k}\right)$ and such that the subgraph induced by $W_{i}$ is a clique for $i=1, \ldots, k$. Note that every clique partitioning $A$ induces a unique partition $W_{1}, \ldots, W_{k}$ of $V$ such that $A=$ $E\left(W_{1}, \ldots, W_{k}\right)$. In case $G$ is complete every partition of the node set of $G$ induces a clique partitioning.

A cycle $C \subseteq E$ of length $k$ is an edge set of the form $\left\{v_{1} v_{2}, v_{2} v_{3}, \ldots, v_{k-1} v_{k}, v_{1} v_{k}\right\}$, where $v_{i} \neq v_{j}$ if $i \neq j$. For $k \geqslant 4$ the set

$$
\bar{C}:=\left\{v_{i} v_{i+2} \mid i=1, \ldots, k-2\right\} \cup\left\{v_{1} v_{k-1}, v_{2} v_{k}\right\}
$$

is called the set of 2-chords of $C$.

We introduce now some basic concepts from polyhedral theory needed in the sequel-see Schrijver (1986) for a comprehensive treatment of this subject.

Our "universe" will be the vector space $\mathbb{R}^{E}$, where $E$ is the edge set of a graph. 
If $F \subseteq E$ then $\chi^{F} \in \mathbb{R}^{E}$ denotes the incidence vector of $F$, that is, $\chi_{e}^{F}=1$ if $e \in F$, $\chi_{e}^{F}=0$ otherwise. We denote the convex hull of a set $S \subseteq \mathbb{B}^{E}$ by $\operatorname{conv}(S)$.

A polytope $P$ is the convex hull of finitely many points, or equivalently, a bounded set that is the intersection of finitely many halfspaces. An inequality $a^{\mathrm{T}} x \leqslant \alpha$ is valid with respect to $P$ if $P \subseteq\left\{x \mid a^{\mathrm{T}} x \leqslant \alpha\right\}$. If $a^{\mathrm{T}} x \leqslant \alpha$ is valid with respect to $P$ then $F_{a}:=\left\{x \in P \mid a^{\mathrm{T}} x=\alpha\right\}$ is the face induced by $a^{\mathrm{T}} x \leqslant \alpha$. A facet of $P$ is a face of $P$ that is contained in no other face of $P$ different from $P$. Equivalently, a facet $F$ of $P$ is a nonempty face with $\operatorname{dim}(F)=\operatorname{dim}(P)-1$, where $\operatorname{dim}(S)$ denotes the dimension of a set $S$, i.e., the maximum number of affinely independent points in $S$ minus 1 . If $P \subseteq \mathbb{R}^{E}$ has dimension $|E|$ then every facet of $P$ is induced by a valid inequality $a^{\mathrm{T}} x \leqslant \alpha$ that is unique up to multiplication by a positive constant. If $a^{\mathrm{T}} x \leqslant \alpha$ is valid for $P$ and $F_{a}:=P \cap\left\{x \mid a^{\mathrm{T}} x=\alpha\right\}$ is a facet of $P$ we say that $a^{\mathrm{T}} x \leqslant \alpha$ is facet-defining.

If $x \in \mathbb{R}^{E}$ and $F \subseteq E$ then the sum $\sum_{e \in F} x_{e}$ is abbreviated by $x(F) .|S|$ denotes the cardinality of a set $S$, and, for $\alpha \in \mathbb{R},\lfloor\alpha\rfloor$ denotes the largest integer not larger than $\alpha$.

\section{Clustering, aggregation, clique partitioning}

The generic clustering problems we are considering in this paper are most frequently stated in the following (imprecise) form:

Given $n$ objects and $p$ characteristics and an $n \times p$ data matrix $D=\left(d_{i k}\right)$, where an entry $d_{i k}$ represents the property of object $i$ with respect to characteristic $k$, find a "best" partition of the set of objects into nonoverlapping classes of "homogeneous" objects, i.e., find a best clustering of the objects.

Some of the terms above need explanation. The data matrix $D$ may contain numerical entries but-in the area of applications we have in mind-it is not the numerical values that are important. We view a column of $D$ as a way to describe which of the objects has which property of the characteristic. For instance, if the objects are "people" and the characteristic is "color of hair" then the entries could be "black", "brown", "red" etc., or if the objects are "voters" and the characteristic is a certain "motion" then one would use the numbers $0,1,2$ and 3 to indicate whether a voter was in favor, against, abstained, or absent. (So the data are nominal and that is why this area is often called qualitative data analysis.) Another natural way to represent such a problem is, thus, to associate with every characteristic $k$ a binary relation $R_{k}$ (which represents the "similarity" between the object pairs $i, j$ ) by defining

$$
(i, j) \in R_{k} \Leftrightarrow d_{i k}=d_{j k} .
$$

Even if exact numerical data are available it is sometimes reasonable to "condense" these data into binary relations. One way, for instance, is to group objects according 
to their "size", e.g., states, companies, or mammals are classified as small, medium, or large (or whatever seems appropriate for the application considered).

There are several ways to interpret the terms "best" and "homogeneous" used in (2.1). Having made the step into the language of binary relations it is most natural to say that a "partition of the objects into nonoverlapping classes of "homogeneous" objects" is nothing but an equivalence relation on the set of objects. So the clusters of a clustering are exactly the equivalence classes defined by the equivalence relation. And a natural way to define "best" is to compare each equivalence relation $R$ with each of the given binary relations $R_{k}$, i.e., to count the number of disagreements of $R$ with $R_{k}$ (this is the number $\left|R \Delta R_{k}\right|:=\mid\left\{(i, j):(i, j) \in R\right.$ and $(i, j) \notin R_{k}$, or $(i, j) \notin R$ and $\left.(i, j) \in R_{k}\right\} \mid$ ), add up the number of disagreements over all given relations, and choose the best equivalence relation with respect to this criterion. Using these interpretations, the clustering problem (2.1) can then be reformulated (precisely) as follows.

Given $p$ binary relations $R_{1}, \ldots, R_{p}$ defined on a set $N$, find an equivalence relation $R^{*}$ on $N$ such that $\sum_{k=1}^{p}\left|R^{*} \Delta R_{k}\right|$ is as small as possible.

Problem (2.2) is a classical problem in the area of qualitative data analysis. It is a special type of the so-called problems of aggregation of binary relations and has been investigated intensively (cf. Barthélemy and Monjardet (1981), Marcotorchino and Michaud (1980, 1981a, 1981b), Opitz and Schader (1984), and Tüshaus (1983)). In the sequel we show how it can be reduced to a $0 / 1$ programming problem.

Every binary relation on a set $N$ can be represented by a $0 / 1$-matrix as follows. For every $k, 1 \leqslant k \leqslant p$, and every pair $(i, j) \in N \times N$ we let

$$
r_{i j}^{(k)}:= \begin{cases}1 & \text { if }(i, j) \in R_{k} \\ 0 & \text { otherwise. }\end{cases}
$$

Similarly, we let

$$
r_{i j}:= \begin{cases}1 & \text { if }(i, j) \in R, \\ 0 & \text { otherwise. }\end{cases}
$$

Then the (nonlinear) objective function $\sum_{k=1}^{p}\left|R \Delta R_{k}\right|$ can be linearized as follows:

$$
\begin{aligned}
\sum_{k=1}^{p}\left|R \Delta R_{k}\right| & =\sum_{k=1}^{p} \sum_{(i, j) \in N \times N}\left(r_{i j}^{(k)}-r_{i j}\right)^{2} \\
& =\sum_{k} \sum_{(i, j)} r_{i j}^{(k)}+\sum_{k} \sum_{(i, j)}\left(1-2 r_{i j}^{(k)}\right) r_{i j} \\
& =c+\sum_{(i, j)}\left(\sum_{k}\left(1-2 r_{i j}^{(k)}\right)\right) r_{i j} \\
& =c+\sum_{(i, j)} c_{i j} r_{i j} .
\end{aligned}
$$


That is,

$$
\begin{aligned}
& \sum_{k=1}^{p}\left|R \Delta R_{k}\right|=\sum_{(i, j) \in N \times N} c_{i j} r_{i j}+c, \\
& c_{i j}:=\sum_{k=1}^{p}\left(1-2 r_{i j}^{(k)}\right)=p-2\left|\left\{k \in\{1, \ldots, p\}:(i, j) \in R_{k}\right\}\right|
\end{aligned}
$$

and

$$
c:=\sum_{(i, j) \in N \times N}\left|\left\{k \in\{1, \ldots, p\}:(i, j) \in R_{k}\right\}\right|
$$

It is also easy to express the requirement that a binary relation $R$ has to be reflexive, symmetric, and transitive by means of equations and inequalities involving the $r_{i j}$ and hence problem (2.2) can be formulated as

$$
\begin{array}{lll}
\text { minimize } & \sum_{(i, j) \in N \times N} c_{i j} r_{i j}+c & \\
\text { subject to } & r_{i i}=1 \text { for all } i \in N & \text { (i.e., } R \text { is reflexive), } \\
& r_{i j}-r_{j i}=0 \text { for all } i, j \in N, i \neq j & \text { (i.e., } R \text { is symmetric), } \\
& r_{i j}+r_{j k}-r_{i k} \leqslant 1 \text { for all } i, j, k \in N & \text { (i.e., } R \text { is transitive), } \\
& r_{i j} \in\{0,1\} \text { for all } i, j \in N . &
\end{array}
$$

Problern (2.3) can be simplified further. Clearly, we can drop the constant $c$ from the objective function and we can also delete the variables $r_{i i}$ from (2.3). Since $r_{i j}=r_{j i}$ we can replace these two variables by one variable $x_{i j}$ (here the order of $i$ and $j$ is irrelevant, so we may assume $i<j$ ), and by defining new weights $w_{i j}:=c_{i j}+c_{j i}$, we get the following $0 / 1$ linear program:

$$
\begin{array}{cl}
\text { minimize } & \sum_{1 \leqslant i<j \leqslant n} w_{i j} x_{i j} \\
\text { subject to } & x_{i j}+x_{j k}-x_{i k} \leqslant 1 \text { for all } 1 \leqslant i<j<k \leqslant n, \\
& x_{i j}-x_{j k}+x_{i k} \leqslant 1 \text { for all } 1 \leqslant i<j<k \leqslant n, \\
& -x_{i j}+x_{j k}+x_{i k} \leqslant 1 \text { for all } 1 \leqslant i<j<k \leqslant n, \\
& x_{i j} \in\{0,1\} \text { for all } 1 \leqslant i<j \leqslant n,
\end{array}
$$

which is clearly equivalent to (2.3).

It is easy to see that the solutions of (2.4) are exactly the incidence vectors of the clique partitionings of the complete graph $K_{n}=\left(V_{n}, E_{n}\right)$. Thus the clustering problem (2.1), resp. its related theoretical interpretation (2.2), can be reduced to the following 
combinatorial optimization problem (called clique partitioning problem (short: (CPP)):

Given a complete graph $K_{n}=\left(V_{n}, E_{n}\right)$ with weights $w_{e} \in \mathbb{Z}$ for all $e \in E_{n}$, find a clique partitioning $A \subseteq E_{n}$ such that $w(A)$ is as small as possible.

We want to attack the clustering problem (2.2) algorithmically via the clique partitioning problem (2.5). Unfortunately, problems (2.2) and (2.5) are NP-hard, cf. Wakabayashi (1986); so we cannot expect to obtain a polynomial time algorithm. We have chosen to develop a cutting plane algorithm for (2.5). To do this, the polyhedron associated with the clique partitioning problem has to be investigated. This approach will be explained in the following section.

\section{The clique partitioning polytope}

To formulate CPP in polyhedral, respectively linear programming terms, we associate with it a polyhedron in the following way. Let $\mathbb{R}^{E_{n}}$ denote the real vector space where every component $x_{e}$ of a vector $x \in \mathbb{R}^{E_{n}}$ is indexed by an edge $e$ of the complete graph $K_{n}=\left(V_{n}, E_{n}\right)$. To avoid trivialities, we assume throughout the paper that $n \geqslant 3$. For every edge set $A \subseteq E_{n}, \chi^{A} \in \mathbb{R}^{E_{n}}$ denotes its incidence vector. The convex hull of all incidence vectors of clique partitionings of $K_{n}$ is called the clique partitioning polytope (of $K_{n}$ ) and is denoted by $\mathscr{P}_{n}$, i.e.,

$$
\mathscr{P}_{n}=\operatorname{conv}\left\{\chi^{A} \in \mathbb{R}^{E_{n}} \mid A \text { is a clique partitioning of } K_{n}\right\} .
$$

Since the vertices of $\mathscr{P}_{n}$ are in one-to-one correspondence with the clique partitionings of $K_{n}$, it follows immediately that CPP can be formulated as the problem

$$
\begin{array}{ll}
\text { minimize } & w^{\mathrm{T}} x \\
\text { subject to } & x \in \mathscr{P}_{n} .
\end{array}
$$

(3.1) is a linear program in the sense that a linear objective function is to be minimized over a polytope. To apply LP-techniques, this formulation is of no use unless $\mathscr{P}_{n}$ can be represented by a system of linear inequalities. Since the clique partitioning problem (2.5) is NP-hard, it follows from general results of complexity theory that it is very unlikely that an explicit complete description of $\mathscr{P}_{n}$ can ever be obtained. But we were able to determine large classes of valid and facet-defining inequalities for $\mathscr{P}_{n}$. The following theorem is a summary of some of the results of Grötschel and Wakabayashi (1987).

Theorem 3.2. Let $K_{n}=\left(V_{n}, E_{n}\right)$ be a complete graph with $n \geqslant 3$ nodes, and let $\mathscr{P}_{n} \subseteq \mathbb{R}^{E_{n}}$ be the clique partitioning polytope of $K_{n}$.

(a) The dimension of $\mathscr{P}_{n}$ is equal to $\left|E_{n}\right|=n(n-1) / 2$.

(b) For every edge $e \in E_{n}$, the trivial inequalities $x_{e} \geqslant 0$ and $x_{e} \leqslant 1$ are valid for $\mathscr{P}_{n}$. Every inequality $x_{e} \geqslant 0$ defines a facet of $\mathscr{P}_{n}$ but no inequality $x_{e} \leqslant 1$ does. 
(c) For every three different nodes $i, j, k \in V_{n}$, each of the three associated triangle inequalities

$$
x_{i j}+x_{j k}-x_{i k} \leqslant 1, \quad x_{i j}-x_{j k}+x_{i k} \leqslant 1, \quad-x_{i j}+x_{j k}+x_{i k} \leqslant 1,
$$

defines a facet of $\mathscr{P}_{n}$.

(d) For every two disjoint nonempty subsets $S$, $T$ of $V_{n}$, the 2-partition inequality induced by $S$ and $T$ (short: $[S, T]$-inequality)

$$
x([S: T])-x\left(E_{n}(S)\right)-x\left(E_{n}(T)\right) \leqslant \min \{|S|,|T|\}
$$

is valid for $\mathscr{P}_{n}$. It defines a facet of $\mathscr{P}_{n}$ if and only if $|S| \neq|T|$.

(e) For every cycle $C \subseteq E_{n}$ of length at least 5 and its set $\bar{C}$ of 2 -chords, the 2-chorded cycle inequality

$$
x(C)-x(\bar{C}) \leqslant\left\lfloor\frac{|C|}{2}\right\rfloor
$$

is valid for $\mathscr{P}_{n}$. It defines a facet of $\mathscr{P}_{n}$ if and only if $|C|$ is odd.

(f) For every even cycle $C \subseteq E_{n}$ of length at least 8 , for every node $z \in V_{n}$ not in the node set $V_{n}(C)$ of $C$, and for every bipartition $V, \bar{V}$ of $V_{n}(C)$, the 2-chorded even wheel inequality

$$
x(C \cup R)-x(\bar{C} \cup \bar{R}) \leqslant \frac{|C|}{2}
$$

defines a facet of $\mathscr{P}_{n}$, where $\bar{C}$ is the set of 2-chords of $C$ and $R:=\{z v \mid v \in V\}$, $\bar{R}:=\{z v \mid v \in \bar{V}\}$.

The proofs of these results are quite involved. These are not all facets of $\mathscr{P}_{n}$ known-see Grötschel and Wakabayashi (1987) for further details. Let us set

$$
\mathscr{T}_{n}:=\left\{x \in \mathbb{R}^{E_{n}} \mid x \geqslant 0, x \text { satisfies all triangle inequalities } 3.2 \text { (c) }\right\} .
$$

Then, by (3.2), all inequalities defining $\mathscr{T}_{n}$ induce facets of $\mathscr{P}_{n}$ and, by (2.4),

$$
\mathscr{P}_{n}=\operatorname{conv}\left\{x \in \mathscr{T}_{n} \mid x \text { integral }\right\}
$$

So the linear program

$$
\begin{array}{ll}
\operatorname{minimize} & w^{\mathrm{T}} x \\
\text { subject to } & x \in \mathscr{T}_{n},
\end{array}
$$

is an LP-relaxation of the clique partitioning problem. Our computational experiments-see Section 5-show that (3.3) is indeed quite a reasonable LP-relaxation.

\section{The cutting plane algorithm}

The use of polyhedral results in LP-based cutting plane procedures has become a standard (and very successful) technique in the recent years-see, for instance, 
Barahona, Grötschel, Jünger and Reinelt (1988), Crowder and Padberg (1980), Grötschel and Holland (1985), Grötschel, Jünger and Reinelt (1984), Padberg and Rinaldi (1987), Reinelt (1985). Nevertheless, to make the basic idea work, requires some nontrivial, rather problem dependent effort.

We will first describe the fundamentals of our algorithm. Afterwards we go into more detail and explain some implementational aspects and some of the heuristics we have added to the basic algorithm to make it work in practice.

The initial step of the polyhedral approach to combinatorial optimization is to get a very good linear programming relaxation of the problem considered. We believe-and computational experience shows-that the system of inequalities given by the classes of facets described in Theorem 3.2 is such an LP-relaxation. It is, however, far from clear how linear programs over these systems of inequalities can be solved efficiently.

It follows from the ellipsoid method, see Grötschel, Lovász and Schrijver (1981), that a linear program (with possibly exponentially many inequalities) can be solved in polynomial time if the separation problem for the constraint system can be solved in polynomial time. The separation problem here is the task to check, for a given vector $y$, whether $y$ satisfies all constraints, and if not, to find a constraint that is violated by $y$. (Such a constraint is called a cutting plane or cut, for obvious reasons.)

For the classes of facet-defining inequalities given in Theorem 3.2, it is clear that the trivial constraints (b) and the triangle inequalities (c) can be checked in polynomial time. However, we do not know whether the separation problem for any of the other systems of inequalities can be solved in polynomial time. Thus to take care (at least partly) of these inequalities we have to resort to separation heuristics.

Due to these facts we decided to proceed as follows. We first solve (by trivial inspection) the linear program

$$
\begin{array}{ll}
\text { minimize } & w^{\mathrm{T}} x \\
\text { subject to } & 0 \leqslant x_{e} \leqslant 1 \text { for all } e \in E_{n} .
\end{array}
$$

Suppose $x^{*}$ is an optimum solution of the present LP. We now check whether $x^{*}$ violates any of the triangle inequalities. If so, we add some of these inequalities (the selection process will be described later) to the current LP, and we repeat.

If $x^{*}$ satisfies all triangle inequalties and is integral we know that it is the incidence vector of a clique partitioning and stop with an optimum solution of (3.1).

If $x^{*}$ satisfies all triangle inequalities and is fractional there is no obvious way to continue, since no further polynomial time separation algorithm is available. We have thus invented several heuristics that check whether a given point $x^{*}$ violates inequalities of the other classes described in Theorem 3.2. Our final choice of which separation heuristic to use was then based on extensive computational testing. This is not too satisfying from a theoretical point of view, but we do not know a way to get around this kind of numerical experiments. 
The result of these experiments was that we gave up considering the 2-chorded cycle inequalities and the 2-chorded even wheel inequalities (and the further facets we know) completely. We only added three heuristics that search for violated 2-partition inequalities 3.2 (d).

So our algorithm now calls these three heuristics to check whether some 2-partition inequalities violated by $x^{*}$ can be found. If this is so, these inequalities are added to the current LP and we repeat. Otherwise, we resort to branch and bound.

It was more than surprising for us that, in all problems we ran, we never had to call the branch and bound algorithm. The cutting plane phase always ended with an integral optimum solution. So the chosen LP-relaxation of (3.1) consisting of all inequalities 3.2 (b) and (c) and some of the inequalities 3.2 (d) turned out to be (empirically) very effective.

Let us now describe a few more details to show our strategic and tactical choices.

LP-solver: Of couse, we did not use the ellipsoid method. We solved our linear programs with IBM's LP-package MPSX/370. This is quite a fast LP-solver, but it is not so easy to use it in a cutting plane environment. Anyway, MPSX did its job. The very first LP was solved by inspection. In all subsequent problems we first called the routine DUAL to find a feasible solution (based on the optimum basis of the previous LP), and then we called PRIMAL to obtain an optimum solution.

Variable elimination: The first issue to think of is whether one intends to eliminate variables in order to run a sparse subproblem. In this case one has to write a routine that brings in the left out variables, prices them out, and restarts the whole program on a larger set of variables in case some reduced costs have the wrong sign. The largest real world problem we got is a clustering problem for 158 objects. So this gives a clique partitioning problem with 12403 variables. This size is around the break even point where pricing out as described above starts to pay (an observation made in other cutting plane experiments). Thus we decided to drop that option and run on the full variable set.

Checking triangle inequalities: There are $3\left(\begin{array}{l}n \\ 3\end{array}\right)$ triangle inequalities 3.2 (c). Based on the problem dimensions we were going for, we decided that complete enumeration of all the inequalities would be feasible with respect to running time. It turned out, however, that, by checking all triangle inequalities, sometimes several thousand violated inequalities were found. To keep the LP's small we decided to add not all of them to the current LP. After some experiments (more on that in Section 5) we adopted the following strategy. First, we introduced a parameter, called MAXCUT, to limit the number of cutting planes added in one iteration. In addition, the enumeration process was organized in such a way that, as soon as about $5 \times$ MAXCUT violated triangle inequalities are found, the enumeration terminates. Moreover, for each violated triangle inequality $a^{\mathrm{T}} x \leqslant 1$ we compute its "degree of violation", i.e., the number $\delta_{a}:=a^{\mathrm{T}} x^{*}-1$, and sort these numbers into 10 buckets 
of equal size. After termination of this procedure we start retrieving violated triangle inequalities from the buckets (starting with the bucket of highest degree of violation) until MAXCUT inequalities have been chosen or all buckets are empty. This way we generate a "reasonable" number of cutting planes all of which are "highly" violated. Limited computational experiments showed that

$$
\text { MAXCUT } E\{400,500\}
$$

is a good choice for our range of problem sizes.

Note that by considering triangle inequalities first and handling them in the way described above yields (empirically) the following benefits:

- The LP's are kept small and sparse (with considerable numerical and running time advantages).

- The heuristics for other classes of inequalities can use the fact that all triangle inequalities are satisfied.

Separation heuristics for 2-partition inequalities: Here we assume that all triangle inequalities are satisfied. As before, we will not generate more than MAXCUT cutting planes in one phase.

We first run a heuristic that searches for $[S, T]$-inequalities with $|S|=1$. For every node $v \in V_{n}$ we do the following. We set $W:=\left\{w \in V_{n} \backslash\{v\} \mid 0<x_{v w}^{*}<1\right\}$ (the nodes with $x_{v w}^{*} \in\{0,1\}$ will not help in this process) and choose some ordering of the nodes of $W$. We pick the first node $w \in W$ (in this ordering), set $T:=\{w\}$, and for every node $i \in W \backslash\{w\}$ we set

$$
T:=T \cup\{i\} \quad \text { if } x_{i j}^{*}=0 \text { for all } j \in T .
$$

We check whether the set $T$ constructed this way satisfies $x^{*}([\{v\}: T])>1$. If so the inequality $x([\{v\}: T]) \leqslant 1$ is added to the current LP. We repeat this procedure with converse ordering of $W$.

We call the next heuristic only if the previous one failed to produce any violated inequality. The second heuristic is the same as the first, we only replace (4.2) by

$$
T:=T \cup\{i\} \quad \text { if } x_{i v}^{*}-\sum_{j \in T} x_{i j}^{*}>0 .
$$

Both these heuristics have an $\mathrm{O}\left(n^{3}\right)$ worst case running time but are much faster in practice.

Our third routine searches for violated $[S, T]$-inequalities with $|S|,|T| \geqslant 2$. It is a complex enumerative procedure with $O\left(n^{3}\right)$ running time, and we do not want to describe it here. In fact, due to our order of calling the heuristics, it turned out that this last heuristic was never ever called in our applications.

Row elimination: After having determined some new cutting planes and before restarting the LP-solver we eliminate all old constraints that are nonbinding at the current optimum solution, i.e., we delete all those rows $a^{\mathrm{T}} x \leqslant \alpha$ with $a^{\mathrm{T}} x^{*}<\alpha$. It 
may, thus, happen that some cuts are generated several times; but in general, row elimination tends to produce smaller linear programs and an overall running time decrease.

Branch and Bound: If the cutting plane phase does not produce an integral solution a branch and bound procedure is called to find an optimum solution. As the implementation of branch and bound algorithms is well known we shall not elaborate on this. We only want to mention that when a variable is temporarily (resp. permanently) fixed to 1 or 0 then we can use the triangle inequalities to fix temporarily (resp. permanently) other variables. That is, if $x_{a}$ and $x_{b}$ have been fixed to 1 and $\{a, b, c\}$ is a triangle in $K_{n}$, then we can fix $x_{c}$ to 1 . This amounts to finding the transitive closure of the graph defined by the edges $e$ with $x_{e}=1$. As stated before, the branch and bound phase was never activated in the final version of our algorithm.

\section{Applications and computational results}

In this section we report on the computational experiences with our cutting plane algorithm for the clique partitioning problem. This algorithm was primarily developed for the clustering problem (2.1) resp. (2.2) which is - as we showed in Section 2-reducible to CPP. All real world instances of CPP we consider here arise this way.

As mentioned in Section 2 , the $p$ binary relations $R_{k}, 1 \leqslant k \leqslant p$, on the object set $N:=\{1,2, \ldots, n\}$ are defined by

$$
(i, j) \in R_{k} \Leftrightarrow d_{i k}=d_{j k} .
$$

Thus the corresponding instance of CPP consists of a complete graph $K_{n}=\left(V_{n}, E_{n}\right)$ with weights $w_{i j}$ assigned to its edges, where $w_{i j}:=p-2\left|\left\{k \in\{1, \ldots, p\}:(i, j) \in R_{k}\right\}\right|$. In two of the applications to be mentioned in the next section some binary relations (that are based on quantitative data) will be defined in a slightly different way.

In all applications given here the input to our program is the data matrix $D$ whose columns define the given binary relations and an additional parameter which determines how the weights $w_{i j}$ are to be calculated. The output is a list of the objects in each of the equivalence classes (i.e., a list of the clusters) determined by the optimum clique partitioning.

We ran our program on a Siemens 7.865 of the Deutsche Forschungs- und Versuchsanstalt für Luft- und Raumfahrt (DFVLR) in Oberpfaffenhofen under the operating system VM/370-CMS. The CPU time reported here is the figure printed out by the operating system as the execution time for the entire run, including all input and output operations. The fractions of seconds were rounded up.

We have considered many real as well as random data but-as mentioned before-in none of the cases we needed to go into the branch and bound phase. More surprisingly, in most cases triangle inequalities were sufficient to produce an 
integral optimum solution. Only a few times 2-partition inequalities (with $|S|=1$ ) had to be added.

\subsection{The problem instances and the optimization process}

In the sequel we consider various examples of clique partitioning problems to illustrate the performance of our algorithm on real-world problem instances. Most of the data sets correspond to applications from the literature concerning the problem of classifying objects based on their qualitative and/or quantitative description. Some new data sets that have not yet appeared in the literature are also given. The complete data sets of all problems considered, and the corresponding solutions are given in the Appendix.

For each problem instance we have summarized the relevant execution data in a table. All Tables 5.1-5.13 are printed in the same scheme. The symbol "\#" means "number of". The optimization process is shown step by step and the total CPU time for the entire run is given. The column "Iter." indicates the iteration number, where each iteration is one complete cutting plane phase (cutting plane recognition, row elimination, solution of the new LP). Thus, the last number in this column is the number of LP's that had to be solved. The next three columns show how many cuts are generated, added, and eliminated per iteration. In the column "LP size" we give the number of constraints of the current LP-the one obtained after the addition and elimination of the cuts indicated in the previous columns. The last column shows the objective function value for the optimum solution of the current LP. The last value in this column is the objective function value for the optimum integer solution found. As it will be clear from the tables, we have considered MAXCUT $=400$ or $\mathrm{MAXCUT}=500$.

To distinguish between the types of cuts generated, we use the symbol " $\Delta$ " to denote triangle inequalities and "*" to denote $[S, T]$-inequalities with $|S|=1$.

Note that the first row (Iter. $=0$ ) corresponds to the trivial LP (4.1) whose optimum solution is obvious. The cuts generated in iteration $k, k \geqslant 1$, are based on the optimum solution obtained in iteration $k-1$.

\subsubsection{Classification of cetacea (whales, porpoises, and dolphins)}

The problem of classifying animals and plants is of great interest in biology. The first example we want to consider here was proposed by Vescia (1985) and concerns the classification of 36 different types of cetacea. The given instance consists of 35 different genera and one species (the Balaenoptera musculus) which are described with respect to 15 characteristics (10 morphological, 3 osteological and 2 behavioral parameters).

Many different mathematical models, including the one we used, have been proposed for this particular problem instance and slightly different classifications were found. As a matter of fact, the cetacea are still poorly known and different zoologists propose different classifications. 
The computational results we obtained with the application of our cutting plane algorithm to this data set $(n=36, p=15$, \# LP variables $=630)$ are summarized in Table 5.1.

Table 5.1

\begin{tabular}{llllll} 
& \multicolumn{5}{c}{ \#Cuts } \\
Iter. & Generated & Added & Eliminated & LP size & Obj. value \\
\hline 0 & - & - & - & - & -998 \\
1 & $274 \Delta$ & 274 & - & 274 & -967 \\
2 & $12 \Delta$ & 12 & - & 286 & -967
\end{tabular}

CPU time: 0:15 (min:sec)

The information given in Table 5.1 are to be interpreted as follows: At first the trivial LP was solved and an optimum solution, say $x^{0}$, was obtained. The objective function value at $x^{0}$ was found to be -998 . Then, 274 triangle inequalities which were violated by $x^{0}$ were generated, and these were all added to the trivial LP. The new LP with 274 constraints was solved and an optimum solution $x^{1}$ with $w^{\mathrm{T}} x^{1}=$ -967 was obtained. Twelve triangle inequalities violated by $x^{1}$ were generated and these were added to the previous LP yielding a new LP with 286 constraints. The optimum solution of this last LP, with objective function value -967 , was integral and an optimum solution for CPP.

This problem instance has been solved to optimality by Vescia (1985), using the approach of solving the dual version of the LP defined by the triangle inequalities and the nonnegativity constraints. The author, however, does not report any computational details.

Remark. In this particular problem some information is missing (cf. entries "**" in the Table A1 given in the Appendix A1) and for this reason the weights $w_{i j}, i<j$, were calculated as follows:

$$
w_{i j}:=\bar{s}_{i j}-s_{i j}
$$

where

$$
s_{i j}:=\left|\left\{k: d_{i k}=d_{j k}, d_{i k} \neq *, 1 \leqslant k \leqslant p\right\}\right|
$$

and

$$
\bar{s}_{i j}:=\left|\left\{k: d_{i k} \neq d_{j k}, d_{i k} \neq *, d_{j k} \neq *, 1 \leqslant k \leqslant p\right\}\right| .
$$

It is assumed here that when an entry, say $d_{i k}$, in the data matrix is "*" then the object $i$ is not comparable to any of the other objects with respect to the characteristic $k$. Thus $s_{i j}$ (resp. $\bar{s}_{i j}$ ) corresponds to the number of characteristics with respect to 
which the objects $i$ and $j$ have (resp. do not have) the same description, provided they are comparable. Note that in this case $s_{i j}+\bar{s}_{i j} \leqslant p$, but if in particular $s_{i j}+s_{i j}=p$, then $w_{i j}=\bar{s}_{i j}-s_{i j}=p-2 s_{i j}$. That is, when no information is missing we have our usual definition.

\subsubsection{Classification of wild cats}

This problem instance is similar to the previous one. The data set consists of the description of 30 wild cats with respect to 14 morphological and behavioral characteristics (cf. Appendix A2). This example is from Marcotorchino (1981), who uses the same mathematical model but solves it to optimality with another method. Again only two iterations were needed to find an optimal solution and only 555 cutting planes sufficed to prove optimality. (In this instance $n=30, p=14$ and \# LP variables $=435$ ).

Table 5.2

\begin{tabular}{llllll}
\hline & & & & & \\
Iter. & Generated & Added & Eliminated & LP size & Obj. value \\
\hline 0 & - & - & - & - & -1400 \\
1 & $561 \Delta$ & 400 & - & 400 & -1313 \\
2 & $155 \Delta$ & 155 & - & 555 & -1304 \\
\hline
\end{tabular}

CPU time: 0:23 (min:sec)

\subsubsection{Classification of workers}

In this example from Opitz and Schader (1984) the data matrix (given in Appendix A3) describes the opinions of 34 workers with respect to 13 parameters concerning their working environment, colleagues, headman, career perspectives, etc.

We have been informed (private communication) that Opitz and Schader have solved this problem to optimality using a branch and bound method proposed by Tüshaus (1983). (In this example $n=34, p=13$ and \# LP variables $=561$ ).

Table 5.3 shows that-contrary to the previous examples-violated inequalities other than the triangle inequalities had to be added to find an optimum solution. The solution found at iteration 7 satisfies all triangle inequalities but is fractional. Two violated simple 2-partition inequalities were generated at iteration 8 and these were added to the previous LP. The new LP, with 1138 constraints, yielded an optimum solution for CPP. In fact, among the real world problems, this is the only example where inequalities different from triangle inequalities were needed.

\subsubsection{Classification of cars}

In this problem instance 33 cars are described with respect to the frequency repair that was needed for the brake system, fuel system, etc. A total of 13 parameters are 
Table 5.3

\begin{tabular}{|c|c|c|c|c|c|}
\hline \multirow[b]{2}{*}{ Iter. } & \multicolumn{3}{|c|}{ \# Cuts } & \multirow[b]{2}{*}{ LP size } & \multirow[b]{2}{*}{ Obj. value } \\
\hline & Generated & Added & Eliminated & & \\
\hline 0 & - & - & - & - & -1233.0 \\
\hline 1 & $1849 \Delta$ & 400 & - & 400 & -1168.50 \\
\hline 2 & $1481 \Delta$ & 400 & - & 800 & -1074.50 \\
\hline 3 & $1268 \Delta$ & 400 & 173 & 1027 & -1012.83 \\
\hline 4 & $1751 \Delta$ & 400 & 344 & 1083 & -970.00 \\
\hline 5 & $59 \Delta$ & 59 & 39 & 1103 & -965.50 \\
\hline 6 & $4 \Delta$ & 4 & 10 & 1097 & -964.50 \\
\hline 7 & $66 \Delta$ & 66 & 26 & 1137 & -96450 \\
\hline 8 & $2 *$ & 2 & 1 & 1138 & -964.00 \\
\hline
\end{tabular}

CPU time: $3: 18$ (min:sec)

considered (cf. Appendix A4) and the entries are 0 and 1 (where " 1 " stands for greater than average frequency of repair). This data set is given in Hartigan (1975) as a trial data set for an algorithm to construct a tree-a special clustering structure. The results are therefore, not comparable. (We have $n=33, p=13$ and \# LP variables $=528$ ).

Table 5.4.

\begin{tabular}{|c|c|c|c|c|c|}
\hline \multirow[b]{2}{*}{ Iter. } & \multicolumn{3}{|c|}{ \# Cuts } & \multirow[b]{2}{*}{ LP size } & \multirow[b]{2}{*}{ Obj. value } \\
\hline & Generated & Added & Eliminated & & \\
\hline 0 & - & - & - & - & -1748.00 \\
\hline 1 & $1507 \Delta$ & 400 & - & 400 & -1648.00 \\
\hline 2 & $1264 \Delta$ & 400 & - & 800 & -1557.50 \\
\hline 3 & $757 \Delta$ & 400 & 55 & 1145 & -1502.00 \\
\hline 4 & $30 \Delta$ & 30 & 17 & 1158 & -1501.00 \\
\hline
\end{tabular}

CPU time: $2: 35$ (min:sec)

\subsubsection{Classification of micro computers}

The data set considered here is from Chah (1985) and concerns the description of 40 micro computers with respect to 14 characteristics (see Appendix A5).

To deal with some quantitative values (describing price, size of random access memory, etc.) in this particular case we defined the binary relations $R_{k}, 1 \leqslant k \leqslant 14$, as follows:

$$
(i, j) \in R_{k} \Leftrightarrow \frac{\left|d_{i k}-d_{j k}\right|}{\max \left\{d_{i k}, d_{j k}, 1\right\}} \leqslant 0.3 .
$$

The model used by Chah is different from the one we use, and leads to a different result. 
Table 5.5

\begin{tabular}{|c|c|c|c|c|c|}
\hline \multirow[b]{2}{*}{ Iter. } & \multicolumn{3}{|c|}{ \# Cuts } & \multirow[b]{2}{*}{ LP size } & \multirow[b]{2}{*}{ Obj. value } \\
\hline & Generated & Added & Eliminated & & \\
\hline 0 & - & - & - & - & -1270 \\
\hline 1 & $1575 \Delta$ & 500 & - & 500 & -1158 \\
\hline 2 & $1434 \Delta$ & 500 & - & 1000 & -1088 \\
\hline 3 & $1185 \Delta$ & 500 & 279 & 1221 & -996 \\
\hline 4 & $1297 \Delta$ & 500 & 305 & 1416 & -966 \\
\hline 5 & $42 \Delta$ & 42 & 116 & 1342 & -966 \\
\hline
\end{tabular}

\subsubsection{Votes of 54 member states of the United Nations Organization}

The example considered here, given by Marcotorchino (1981), describes the votes of 54 Nations on 3 motions presented in a General Assembly of the UNO in 1968 (see Appendix A6). This problem has been solved to optimality by Marcotorchino, using the approach of solving the dual version of the LP-relaxation defined by the triangle and trivial inequalities.

Table 5.6

\begin{tabular}{llllll}
\hline & \multicolumn{5}{c}{ \# Cuts } \\
Iter. & Generated & Added & Eliminated & LP size & Obj. value \\
\hline 0 & - & - & - & - & -918.00 \\
1 & $2004 \Delta$ & 400 & - & 400 & -886.00 \\
2 & $2001 \Delta$ & 400 & - & 800 & -840.00 \\
3 & $2003 \Delta$ & 400 & 251 & 949 & -833.00 \\
4 & $1716 \Delta$ & 400 & 70 & 1279 & -801.00 \\
5 & $19 \Delta$ & 19 & - & 1298 & -798.00 \\
\hline
\end{tabular}

CPU time: 4:30 (min:sec)

\subsubsection{Votes of all member states of the United Nations Organization}

These are new data sets, collected from UNO (1985), concerning the votes of all member states of the United Nations Organization on Resolutions adopted by the 39th General Assembly held at the end 1984 (cf. Appendix A7).

For each of the data sets to be specified in the sequel, we have run our program for the complete data set $(n=158)$ and for a smaller data set $(n<158)$ obtained from the complete one by deleting some States which were "absent".

Case 1: Resolutions 39/119-120-121-Votes on 3 Resolutions concerning the situation of human rights and fundamental freedom in El Salvador, Guatemala, and Chile (cf. Appendix A7.1). 
Table 5.7

\begin{tabular}{|c|c|c|c|c|c|}
\hline \multirow[b]{2}{*}{ Iter. } & \multicolumn{3}{|c|}{ \# Cuts } & \multirow[b]{2}{*}{ LP size } & \multirow[b]{2}{*}{ Obj. value } \\
\hline & Generated & Added & Eliminated & & \\
\hline 0 & - & - & - & - & -12322 \\
\hline 1 & $2001 \Delta$ & 400 & - & 400 & -12298 \\
\hline 2 & $2003 \Delta$ & 400 & - & 800 & -12280 \\
\hline 3 & $2003 \Delta$ & 400 & 30 & 1170 & -12234 \\
\hline 4 & $1653 \Delta$ & 400 & - & 1570 & -12210 \\
\hline 5 & $1302 \Delta$ & 400 & 37 & 1933 & -12207 \\
\hline 6 & $1401 \Delta$ & 400 & - & 2333 & -12197 \\
\hline
\end{tabular}

CPU time: $14: 12$ (min:sec)

Case 1a: $n=158$ and $p=3$ (\# LP variables $=12403$ ). See Table 5.7.

Case $1 b: n=139$ and $p=3$ (\# LP variables =9591). This data set corrsponds to the previous one without the States which were absent in at least one of the three sessions considered. See Table 5.8 .

Table 5.8

\begin{tabular}{|c|c|c|c|c|c|}
\hline \multirow[b]{2}{*}{ Iter. } & \multicolumn{3}{|c|}{ \# Cuts } & \multirow[b]{2}{*}{ LP size } & \multirow[b]{2}{*}{ Obj. value } \\
\hline & Generated & Added & Eliminated & & \\
\hline 0 & - & - & - & - & -11706 \\
\hline 1 & $2008 \Delta$ & 400 & - & 400 & -11664 \\
\hline 2 & $2001 \Delta$ & 400 & - & 800 & -11650 \\
\hline 3 & $2005 \Delta$ & 400 & 40 & 1160 & -11639 \\
\hline 4 & $846 \Delta$ & 400 & - & 1560 & -11613 \\
\hline
\end{tabular}

Table 5.9

\begin{tabular}{|c|c|c|c|c|c|}
\hline \multirow[b]{2}{*}{ Iter. } & \multicolumn{3}{|c|}{ \# Cuts } & \multirow[b]{2}{*}{ LP size } & \multirow[b]{2}{*}{ Obj. value } \\
\hline & Generated & Added & Eliminated & & \\
\hline 0 & - & - & - & - & -73178 \\
\hline 1 & $2592 \Delta$ & 500 & - & 500 & -73139 \\
\hline 2 & $2502 \Delta$ & 500 & - & 1000 & -72886 \\
\hline 3 & $2501 \Delta$ & 500 & 166 & 1334 & -72874 \\
\hline 4 & $236 \Delta$ & 236 & - & 1570 & -72821 \\
\hline 5 & $87 \Delta$ & 87 & 33 & 1624 & -72820 \\
\hline
\end{tabular}

CPU time: 9:47 (min:sec) 
Case 2: Resolution 39/148_-Votes on 15 matters related with nuclear weapons (cf. Appendix A7.2).

Case $2 a: n=158$ and $p=15$ (\# LP variables $=12403$ ). See Table 5.9.

Case $2 b: n=145$ and $p=15$ (\# LP variables =11165). This data set corresponds to the previous one without the States which were absent at least 8 times. See Table 5.10 .

Case 3: Resolution 39/99_Votes on 9 matters related to the United Nations Relief and Works for Palestine Refugees in the Near East (cf. Appendix A7.3). Case $3 a: n=158$ and $p=9$ (\# LP variables $=12403$ ). See Table 5.11.

Table 5.10

\begin{tabular}{|c|c|c|c|c|c|}
\hline \multirow[b]{2}{*}{ Iter. } & \multicolumn{3}{|c|}{ \# Cuts } & \multirow[b]{2}{*}{ LP size } & \multirow[b]{2}{*}{ Obj. value } \\
\hline & Generated & Added & Eliminated & & \\
\hline 0 & - & - & - & $\ldots$ & -72111 \\
\hline 1 & $2506 \Delta$ & 500 & - & 500 & -72076 \\
\hline 2 & $2513 \Delta$ & 500 & - & 1000 & -71880 \\
\hline 3 & $1426 \Delta$ & 500 & - & 1500 & -71861 \\
\hline 4 & $269 \Delta$ & 269 & - & 1769 & -71819 \\
\hline 5 & $87 \Delta$ & 87 & 38 & 1818 & -71818 \\
\hline
\end{tabular}

CPU time: 8:04 (min:sec)

Table 5.11

\begin{tabular}{llllrl}
\hline & & & & & \\
Iter. & Guts & & & \\
\cline { 2 - 5 } & Generated & Added & Eliminated & LP size & Obj. value \\
\hline 0 & - & - & - & - & -73133 \\
1 & $2502 \Delta$ & 500 & - & 500 & -73090 \\
2 & $2501 \Delta$ & 500 & - & 1000 & -73076 \\
3 & $840 \Delta$ & 500 & - & 1500 & -73076 \\
\hline
\end{tabular}

CPU time: 5:27 (min:sec)

Table 5.12

\begin{tabular}{|c|c|c|c|c|c|}
\hline \multirow[b]{2}{*}{ Iter. } & \multicolumn{3}{|c|}{ \# Cuts } & \multirow[b]{2}{*}{ LP size } & \multirow[b]{2}{*}{ Obj, value } \\
\hline & Generated & Added & Eliminated & & \\
\hline 0 & - & - & - & - & -72694 \\
\hline 1 & $2502 \Delta$ & 500 & - & 500 & -72651 \\
\hline 2 & $2501 \Delta$ & 500 & - & 1000 & -72637 \\
\hline 3 & $840 \Delta$ & 500 & - & 1500 & -72637 \\
\hline
\end{tabular}

CPU time: $4: 43$ (min:sec) 
Case 3b: $n=147$ and $p=9$ (\# LP variables 10731 ). This data set corresponds to the previous one without the States which were absent at least 5 times. See Table 5.12.

\subsubsection{Classification of companies}

The problem instance considered here is from Späth (1977) and concerns the description of 137 companies in W. Germany with respect to their need of different groups of employees (cf. Appendix A8). A total of 25 groups of employees are considered (ex. secretaries, programers, engineers, etc.), and for each company, it is indicated whether the corresponding group of employees is needed (" 1 ") or not ("0"). The clustering technique used by Späth fixes the number of clusters a priori and optimizes a different objective function. The computational results are therefore not comparable.

Table 5.13

\begin{tabular}{llllll}
\hline Iter. & Generated & Added & Eliminated & LP size & Obj. value \\
\hline 0 & - & - & - & - & -82625 \\
1 & $2501 \Delta$ & 500 & - & 500 & -82414 \\
2 & $2502 \Delta$ & 500 & - & 1000 & -82188 \\
3 & $2565 \Delta$ & 500 & 182 & 1318 & -82163 \\
4 & $2550 \Delta$ & 500 & 131 & 1687 & -82051 \\
5 & $2502 \Delta$ & 500 & 31 & 2156 & -81897 \\
6 & $2501 \Delta$ & 500 & 51 & 2605 & -81884 \\
7 & $2549 \Delta$ & 500 & - & 3105 & -81829 \\
8 & $2558 \Delta$ & 500 & 86 & 3619 & -81811 \\
9 & $1885 \Delta$ & 500 & - & 4019 & -81802 \\
\hline
\end{tabular}

CPU time: $19: 47$ (min:sec)

Although the optimum solution we obtained turned out to be not interesting (only two clusters were produced), the computational results reveal some interesting aspects (cf. Table 5.13). Note that the LP at iteration 8 has 3619 constraints, all of which are binding for the obtained solution $\bar{x}$ (this can be derived from the fact that at iteration 9 no elimination was performed). Although there were 1885 triangle inequalities violated by $\bar{x}$, the addition of only 500 of them was sufficient to produce an optimum solution. It should also be noted that in this case the final LP has more than 4000 constraints. This problem is the "hardest" real world problem we encountered so far.

\subsection{Random data}

In the applications we considered previously, only in one case $(n=34)$ we needed to add cuts other than the triangle inequalities. For randomly generated data sets, 
which we created to test various features of our code the picture was, however, different.

We generated 20 matrices with entries $0,1,2$, having dimension $n \times p$ where $12 \leqslant n \leqslant 30,10 \leqslant p \leqslant 13$, and in 9 cases we needed to call the cut generation routine for $[S, T]$-partition inequalities with $|S|=1$. In 7 cases the first strategy used in this routine was sufficient to find violated $[S, T]$-partition inequalities, and in 2 of them we had to use the second strategy (cf. Section 4). In none of the cases we needed to call the third cut generation heuristic for $[S, T]$-inequalities and in all cases (except in one) the running times were less than 2 minutes.

In Table 5.14 we show the computational result obtained for $n=22$ and $p=13$. Observe that when all triangle inequalities are satisfied the objective function value $(-138.50)$ is very close to the optimum objective value $(-137)$. This fact could be observed in all the 7 cases (only in one case the relative difference was $5 \%$, in 6

Table 5.14

$n=22$

\begin{tabular}{rcccc}
\hline \multicolumn{5}{c}{ \# Cuts Generated } \\
\cline { 3 - 4 } Iter. & $\Delta$ & $*(1)$ & $*(2)$ & Obj. value \\
\hline 0 & - & - & - & -237.00 \\
1 & 432 & - & - & -138.50 \\
2 & - & 2 & - & -138.00 \\
3 & 62 & - & - & -138.00 \\
4 & - & 1 & - & -137.75 \\
5 & - & 1 & - & -137.33 \\
6 & 3 & - & - & -137.17 \\
7 & - & 1 & - & -137.11 \\
8 & - & 3 & - & -137.00 \\
9 & 9 & - & - & -137.00 \\
10 & - & - & 2 & -137.00 \\
11 & 13 & - & - & -137.00 \\
\hline
\end{tabular}

CPU time: $1: 21$ (min:sec)

Table 5.15

$n=30$

\begin{tabular}{lllll}
\hline Data & Entries & Cuts & MAXCUT & CPU time \\
\hline Random-1 & $\{0,1,2\}$ & $\Delta *$ & 400 & $0: 23$ \\
Random-2 & $\{0,1,2\}$ & $\Delta *$ & 400 & $0: 15$ \\
Random-3 & $\{0,1,2\}$ & $\Delta$ & 400 & $3: 46$ \\
Random-4 & $\{0,1,2,3\}$ & $\Delta *$ & 400 & $0: 11$ \\
Random-5 & $\{0,1\}$ & $\Delta$ & 400 & $4: 20$ \\
Random-6 & $\{0,1\}$ & $\Delta$ & 400 & $2: 40$ \\
\hline
\end{tabular}


cases it was less than $2 \%$ ). The symbols "*(1)" and "*(2)" stand for $[S, T]$ inequalities found by the first and second strategy, respectively.

The computational results obtained for 6 data matrices of dimension $30 \times p$, where $10 \leqslant p \leqslant 13$, are shown in Table 5.15.

\subsection{Some remarks}

We have tested to which extent the choice of the value for MAXCUT influences the overall running time and we have noted that in most of the cases the better performances were achieved for MAXCUT $\in\{400,500\}$, for $n$ up to 60. Especially when there are many violations the running times may depend largely on the choice of MAXCUT. In these cases, if MAXCUT is less than 300 the improvement on the objective value is very slow. On the other hand, if MAXCUT is too large (say more than 800) the LP's become large very fast, even with eliminations, and they require a lot of time to be optimized. In Table 5.16 we indicate the results obtained for $n=34$ (1849 violations found in the first iteration) and $n=33$ (1507 violations).

Table 5.16

\begin{tabular}{llll}
\hline$n=34$ (workers) & & \multicolumn{1}{l}{$n=33$ (cars) } \\
\cline { 3 - 4 } MAXCUT & CPU time & MAXCUT & CPU time \\
\hline 250 & $4: 40$ & 300 & $2: 45$ \\
400 & $3: 18$ & 400 & $2: 35$ \\
500 & $3: 30$ & 500 & $3: 25$ \\
600 & $4: 13$ & 800 & $3: 36$ \\
\hline
\end{tabular}

By comparing the running times for problem instances with about the same size we observed considerable variances. A closer look at the "structures" of the objective function showed that "easy" problems often have the property that more than $80 \%$ of the objective function coefficients are positive (or negative), while the problems with a more even distribution of positive and negative objective function coefficients needed more time for their solution. More exactly, what matters is the distribution of the $w_{i j}$ in the interval $[-p, p]$. But we do not have sufficient material to derive significant statistical conclusions from our impression.

In Table 5.17 we summarize the computational results obtained for all real data and some random data we have mentioned previously. It is an interesting fact that for the real data sets, except for the case $n=34$, the triangle inequalities were sufficient to obtain an optimum solution. In all cases in which we needed to add cuts other than the triangle inequalities, we obtained that the LP-relaxation defined by the triangle inequalities produced a very good lower bound for the optimum objective value is very slow. On the other hand, if MAXCUT is too large (say more 
Table 5.17

\begin{tabular}{|c|c|c|c|c|c|c|}
\hline$n$ & Data & $\begin{array}{l}\text { type of } \\
\text { cuts }\end{array}$ & $\#$ iter. & $\begin{array}{l}\max \\
\text { LP size }\end{array}$ & $\begin{array}{l}\max \\
\text { \# viol. }\end{array}$ & $\begin{array}{l}\text { CPU time } \\
\text { (min:sec) }\end{array}$ \\
\hline 12 & Random & $\Delta *$ & 4 & 35 & 29 & $0: 11$ \\
\hline 15 & Random & $\Delta$ & 2 & 422 & 357 & $0: 16$ \\
\hline 20 & Random & $\Delta *$ & 3 & 364 & 329 & $0: 31$ \\
\hline 25 & Random & $\Delta$ & 7 & 660 & 741 & $1: 45$ \\
\hline 30 & Random-1 & $\Delta *$ & 7 & 142 & 94 & $0: 23$ \\
\hline 30 & Random-2 & $\Delta *$ & 4 & 96 & 57 & $0: 15$ \\
\hline 30 & Random-3 & $\Delta$ & 4 & 955 & 1016 & $3: 46$ \\
\hline 30 & Random-4 & $\Delta *$ & 2 & 33 & 31 & $0: 11$ \\
\hline 30 & Random-5 & $\Delta$ & 4 & 945 & 1406 & $4: 20$ \\
\hline 30 & Random- 6 & $\Delta$ & 4 & 1206 & 1578 & $2: 47$ \\
\hline 30 & Wild Cats & $\Delta$ & 2 & 555 & 561 & $0: 23$ \\
\hline 33 & Cars & $\Delta$ & 4 & 1158 & 1507 & $2: 35$ \\
\hline 34 & Workers & $\Delta *$ & 8 & 1138 & 1849 & $3: 18$ \\
\hline 36 & Cetacea & $\Delta$ & 2 & 286 & 274 & $0: 15$ \\
\hline 40 & Micro & $\Delta$ & 7 & 1342 & 1575 & $4: 17$ \\
\hline 54 & UNO & $\Delta$ & 5 & 1298 & 2004 & $4: 30$ \\
\hline 60 & Random & $\Delta *$ & 16 & 912 & 742 & $6: 08$ \\
\hline 158 & UNO-1a & $\Delta$ & 6 & 2333 & 2003 & $14: 12$ \\
\hline 139 & UNO-1b & $\Delta$ & 4 & 1560 & 2008 & $8: 38$ \\
\hline 158 & UNO-2a & $\Delta$ & 5 & 1624 & 2592 & $9: 47$ \\
\hline 145 & UNO-2b & $\Delta$ & 5 & 1818 & 2513 & $8: 04$ \\
\hline 158 & UNO-3a & $\Delta$ & 3 & 1500 & 2502 & $5: 27$ \\
\hline 147 & UNO-3b & $\Delta$ & 3 & 1500 & 2502 & $4: 43$ \\
\hline 137 & Companies & $\Delta$ & 9 & 4019 & 2558 & $19: 47$ \\
\hline
\end{tabular}

branch and bound phase, if needed. Surprisingly, in none of the problem instances we needed to enter the branch and bound phase. In fact, we never called our third cut generation routine for general 2-partition inequalities.

The present form of our algorithm should not be seen as a definitive one. It would be interesting to test some other strategies to see whether the running time can be speeded up. Thus for example in the case of the violated triangle inequalities, instead of using our MAXCUT most violated inequalities strategy, one could try to add only variable disjoint triangle inequalities.

Another possibility would be to consider again some other classes of facet-defining inequalities, develop better separation heuristics and call them in different orders. These features could be added to the present code without requiring any substantial modification.

\subsection{Conclusions}

Other codes for the solution of the clustering problem considered here exist-see Marcotorchino and Michaud (1980, 1981a, 1981b), Schader and Tüshaus (1985), or Tüshaus (1983). Unfortunately, we were not able to get hold of any of these codes. So we were not able to execute these algorithms in the same environment in 
order to compare running times, etc. It is also very hard to draw conclusions from the published results of other authors since the performances of the codes are often not documented too well and, even if so, it is unclear how to filter out the performance characteristics of the computers and operating systems used. Anyway, we believe that our approach is a valid alternative to the existing methods. This opinion, in particular, is confirmed by the fact that the running times of our code are quite modest and that it can handle large problem sizes consistently well. Moreover, as far as we know no other code has ever solved clustering problems of the size we report about here.

\section{Appendix}

We list in the sequel either the complete data sets or provide references to papers where the data of the problem instances mentioned in Chapter 5 can be found. The optimum solution we found is listed under the heading "Solution Classes" (SC), where we indicate the class to which each object belongs. Thus, in the column "SC", objects with the same number are to be interpreted as belonging to the same class.

\section{Appendix A1. Classification of cetecea}

Reference: Vescia (1985). The parameters and entries in Table A1 are specified as follows:

(i) Morphological parameters.

1: Neck.

0 : does not exist, 1: exists.

2: Form of the head.

0 : cylindrical, 1 : conical, 2 : with a curved forehead, 3 : globular, 4: flat, 5: convex.

3: Size of the head.

0 : very big, 1 : medium size.

4: Beak.

0: missing, 1: large, 2: narrow and short, 3: narrow and long.

5: Dorsal fin.

0: missing, 1: triangular, 2: falciform, 3: backward and falciform.

6: Flippers.

0 : small, 1: large and short, 2: medium size, 3: long and narrow.

7: Set of teeth.

0 : on the lower jaw, 1: on the lower and upper jaw, 2: without teeth but long baleens, 3: without teeth but thick baleens, 4 : without teeth but large baleens.

9: Blow hole.

0 : on the left side, 1: on the right side, 2 : on the middle line, $3:$ on the middle line with two holes.

10: Color.

0 : central parts are clearer than dorsal parts, 1: blackish, 2: no pigmentation, 3: spotted.

14: Longitudinal furrows on the throat.

0 : do not exist, 1: a small number exists, 2 : a big number exists.

(ii) Osteological parameters.

11: Cervical vertebrae.

0 : free, 1 : partly or completely welded.

12: Lachrymal and jugal bones.

0 : form one piece, 1 : are independent. 


\section{5: Head bones.}

0 : symmetrical, 1: slightly unsymmetrical, 2 : unsymmetrical, 3 : very unsymmetrical.

(iii) Behavioral parameters.

\section{8: Feeding.}

0: feed on squish, 1: feed on fish, 2: feed on seal, 3: feed on plankton.

\section{3: Habitat.}

0 : rivers, 1: temperate or warm seas, 2: cold seas, 3: coasts, 4: variable.

\section{Table A1}

Data matrix and solution classes (SC)

\begin{tabular}{|c|c|c|c|c|c|c|c|c|c|c|c|c|c|c|c|c|c|}
\hline & Zoological name & 1 & 2 & 3 & 4 & 5 & 6 & 7 & 8 & 9 & 10 & 11 & 12 & 13 & 14 & 15 & $\mathrm{SC}$ \\
\hline 1 & Balaena & 0 & 5 & 0 & 0 & 0 & 1 & 2 & 3 & 3 & 4 & 1 & $*$ & 2 & 0 & 0 & 1 \\
\hline 2 & Balaenoptera & 0 & 4 & 0 & 0 & 3 & 0 & 4 & 3 & 3 & 0 & 0 & $*$ & 4 & 2 & 0 & 2 \\
\hline 3 & Balaenoptera Mus. & 0 & 4 & 0 & 0 & 3 & 3 & 4 & 3 & 3 & 3 & 0 & $*$ & 4 & 2 & 0 & 2 \\
\hline 4 & Berardius & 1 & 2 & 1 & 2 & 2 & 0 & 0 & 0 & 2 & 0 & 1 & 1 & 2 & 1 & 2 & 3 \\
\hline 5 & Cephalorhynchus & 0 & 1 & 1 & 1 & 2 & 2 & 1 & 0 & 1 & 4 & 1 & 1 & 2 & 0 & 1 & 4 \\
\hline 6 & Delphinapterus & 1 & 3 & 1 & 0 & 0 & 1 & 1 & 1 & 1 & 2 & 0 & 1 & 2 & 0 & 2 & 5 \\
\hline 7 & Delphinus & 0 & 2 & 1 & 2 & 2 & 2 & 1 & 1 & 1 & 0 & 1 & 1 & 1 & 0 & 1 & 4 \\
\hline 8 & Eschrichtius & 0 & 1 & 0 & 0 & 0 & 3 & 3 & 3 & 3 & 3 & 0 & * & 4 & 1 & 0 & 2 \\
\hline 9 & Eubalaena & 0 & 5 & 0 & 0 & 0 & 1 & 2 & 3 & 3 & 1 & 1 & $*$ & 2 & 0 & 0 & 1 \\
\hline 10 & Globicephala & 0 & 3 & 1 & 0 & 2 & 3 & 1 & 0 & 1 & 3 & 1 & 1 & 1 & 0 & 1 & 4 \\
\hline 11 & Grampus & 0 & 3 & 1 & 0 & 2 & 3 & 0 & 0 & 1 & 0 & 1 & 1 & 4 & 0 & 1 & 4 \\
\hline 12 & Hyperoodon & 1 & 2 & 1 & 2 & 2 & 0 & 0 & 0 & 2 & 0 & 1 & 1 & 4 & 1 & 2 & 3 \\
\hline 13 & Inia & 1 & 2 & 1 & 3 & 1 & 1 & 1 & 1 & 2 & 0 & 0 & 0 & 0 & 0 & $*$ & 6 \\
\hline 14 & Kogia & 0 & 0 & 0 & 0 & 1 & 0 & 0 & 0 & 0 & 0 & 1 & $*$ & 4 & 0 & 3 & 7 \\
\hline 15 & Lagenorhynchus & 0 & 2 & 1 & 2 & 2 & 2 & 1 & 1 & 1 & 0 & 1 & 1 & 2 & 0 & 1 & 4 \\
\hline 16 & Lipotes & 1 & 2 & 1 & 3 & 1 & 1 & 1 & 1 & 0 & 0 & 0 & 0 & 0 & 0 & $*$ & 6 \\
\hline 17 & Lissodelphis & 0 & 1 & 1 & 1 & 0 & 2 & 1 & 1 & 1 & 0 & 1 & 1 & 1 & 0 & 1 & 4 \\
\hline 18 & Megaptera & 0 & 4 & 0 & 0 & 3 & 3 & 4 & 3 & 3 & 3 & 0 & * & 4 & 2 & 0 & 2 \\
\hline 19 & Mesoplodon & 1 & 1 & 1 & 2 & 2 & 0 & 0 & 0 & 2 & 0 & 1 & 1 & 4 & 1 & 2 & 3 \\
\hline 20 & Monodon & 1 & 3 & 1 & 0 & 0 & 1 & 0 & 0 & 1 & 3 & 0 & 1 & 2 & 0 & 2 & 5 \\
\hline 21 & Neopbalaena & 0 & 1 & 0 & 0 & 3 & 0 & 2 & 3 & 3 & 0 & 1 & * & 2 & 0 & 0 & 1 \\
\hline 22 & Neophocaena & 0 & 1 & 1 & 0 & 0 & 0 & 1 & 1 & 1 & 3 & 1 & 1 & 4 & 0 & 1 & 4 \\
\hline 23 & Orcaella & 1 & 3 & 1 & 0 & 2 & 0 & 1 & 1 & 1 & 1 & 1 & 1 & 3 & 0 & 1 & 4 \\
\hline 24 & Orcinus & 0 & 3 & 1 & 0 & 2 & 1 & 1 & 2 & 1 & 3 & 1 & 1 & 2 & 0 & 1 & 4 \\
\hline 25 & Phocaena & 0 & 1 & 1 & 0 & 1 & 0 & 1 & 1 & 1 & 0 & 1 & 1 & 4 & 0 & 1 & 4 \\
\hline 26 & Physeter & 0 & 0 & 0 & 0 & 0 & 0 & 0 & 0 & 0 & 0 & 1 & 0 & 1 & 0 & 3 & 7 \\
\hline 27 & Platanista & 1 & 2 & 1 & 3 & 1 & 1 & 1 & 1 & 2 & 1 & 0 & 0 & 0 & 0 & 1 & 6 \\
\hline 28 & Pseudorca & 0 & 3 & 1 & 0 & 2 & 3 & 1 & 0 & 1 & 1 & 1 & 1 & 4 & 0 & 1 & 4 \\
\hline 29 & Sotalia & 0 & 2 & 1 & 2 & 2 & 2 & 1 & 1 & 1 & 2 & 1 & 1 & 3 & 0 & 1 & 4 \\
\hline 30 & Sousa & 0 & 2 & 1 & 2 & 2 & 2 & 1 & 1 & 1 & 0 & 1 & 1 & 3 & 0 & 1 & 4 \\
\hline 31 & Stenella & 0 & 2 & 1 & 2 & 2 & 2 & 1 & 1 & 1 & 3 & 1 & 1 & 4 & 0 & 1 & 4 \\
\hline 32 & Steno & 0 & 1 & 1 & 2 & 2 & 2 & 1 & 1 & 1 & 0 & 1 & 1 & 1 & 0 & 1 & 4 \\
\hline 33 & Stenodelphis & 1 & 2 & 1 & 3 & 1 & 1 & 1 & 1 & 2 & 1 & 0 & 0 & 0 & 0 & * & 6 \\
\hline 34 & Tasmacetus & 0 & 3 & 1 & 2 & 2 & 0 & 0 & 0 & 2 & 0 & 1 & 1 & 2 & 1 & 2 & 3 \\
\hline 35 & Tursiops & 0 & 2 & 1 & 2 & 2 & 2 & 1 & 1 & 1 & 0 & 1 & 1 & 4 & 0 & 1 & 4 \\
\hline 36 & Ziphius & 0 & 1 & 1 & 2 & 2 & 0 & 0 & 0 & 2 & 3 & 1 & 1 & 4 & 1 & 2 & 3 \\
\hline
\end{tabular}

The missing data are represented by "*”. 


\section{Appendix A2. Classification of wild cats}

Reference: Marcotorchino (1981). The parameters and entries in Table A2 are specified as follows:

(i) Morphological parameters.

1: Aspect of the pelt.

1: without spots, uniformly colored, 2: with spots, 3: with stripes, 4: marmoreal (like marble).

2: Fur.

0: short-haired, 1: long-haired.

3: Ears.

1: round or rounded, 2 : pointed.

4: Height (H) up to shoulder.

1: $H \leqslant 50 \mathrm{~cm}, 2: 50 \mathrm{~cm}<H \leqslant 70 \mathrm{~cm}, 3: H>70 \mathrm{~cm}$.

5: Weight (W).

1: $W \leqslant 10 \mathrm{~kg}, 2: 10 \mathrm{~kg}<W \leqslant 80 \mathrm{~kg}, 3: W>80 \mathrm{~kg}$.

6: Length $(L)$ of body.

1: $L \leqslant 80 \mathrm{~cm}, 2: 80 \mathrm{~cm}<L \leqslant 150 \mathrm{~cm}, 3: L>150 \mathrm{~cm}$.

7: Length of tail compared with length of body,

1: short, 2: median, 3: long.

8: (Teeth) Canines.

0 : little developed, 1: very developed.

9. (Larynx) Lingual bone.

0: absent, 1: present.

10: Retractile claws.

$0:$ no, 1: yes.

(ii) Behavioral parameters.

11: Predatory behavior.

1: diurnal, 2: diurnal and nocturnal, 3: nocturnal.

12: Type of prey.

1: big prey (antelope, buffalo, etc.), 2: big or small prey, 3: small prey (shrewmouse, little monkey).

13: Climbs trees.

0 : no, 1: yes.

14: Chases after or lies in wait for the prey.

0 : wait, 1: chase.

Table A2

Data matrix and solution classes (SC)

\begin{tabular}{|c|c|c|c|c|c|c|c|c|c|c|c|c|c|c|c|c|}
\hline & Wild cats & 1 & 2 & 3 & 4 & 5 & 6 & 7 & 8 & 9 & 10 & 11 & 12 & 13 & 14 & $\mathrm{SC}$ \\
\hline 1 & Lion & 1 & 0 & 1 & 3 & 3 & 3 & 2 & 1 & 1 & 1 & 1 & 1 & 0 & 1 & 1 \\
\hline 2 & Tigre & 3 & 0 & 1 & 3 & 3 & 3 & 2 & 1 & 1 & 1 & 3 & 1 & 0 & 0 & 1 \\
\hline 3 & Jaguar & 2 & 0 & 1 & 3 & 3 & 2 & 1 & 1 & 1 & 1 & 2 & 1 & 1 & 0 & 2 \\
\hline 4 & Leopard & 2 & 0 & 1 & 3 & 3 & 2 & 2 & 1 & 1 & 1 & 3 & 2 & 1 & 0 & 2 \\
\hline 5 & Once & 2 & 1 & 1 & 2 & 2 & 2 & 3 & 1 & 1 & 1 & 1 & 2 & 1 & 0 & 2 \\
\hline 6 & Guepard & 2 & 0 & 1 & 3 & 2 & 2 & 3 & 0 & 0 & 0 & 1 & 2 & 0 & 1 & 3 \\
\hline 7 & Puma & 1 & 0 & 1 & 2 & 3 & 2 & 3 & 1 & 0 & 1 & 2 & 2 & 1 & 0 & 2 \\
\hline 8 & Panth. Nebuleuse & 4 & 0 & 1 & 2 & 2 & 2 & 3 & 1 & 1 & 1 & 3 & 3 & 1 & 0 & 2 \\
\hline 9 & Serval & 2 & 0 & 2 & 2 & 2 & 2 & 1 & 0 & 0 & 1 & 1 & 3 & 1 & 1 & 4 \\
\hline 10 & Ocelot & 2 & 0 & 1 & 2 & 2 & 2 & 2 & 0 & 0 & 1 & 2 & 3 & 1 & 0 & 4 \\
\hline 11 & Lynx & 2 & 1 & 2 & 2 & 2 & 2 & 1 & 1 & 0 & 1 & 2 & 2 & 1 & 0 & 2 \\
\hline 12 & Caracal & 1 & 0 & 2 & 2 & 2 & 1 & 1 & 0 & 0 & 1 & 2 & 3 & 1 & 1 & 4 \\
\hline 13 & C. Viverrin & 2 & 0 & 1 & 1 & 1 & 2 & 2 & 0 & 0 & 1 & 2 & 3 & 0 & 0 & 4 \\
\hline
\end{tabular}


Table A2-continued

\begin{tabular}{|c|c|c|c|c|c|c|c|c|c|c|c|c|c|c|c|c|}
\hline & Wild cats & 1 & 2 & 3 & 4 & 5 & 6 & 7 & 8 & 9 & 10 & 11 & 12 & 13 & 14 & $\mathrm{SC}$ \\
\hline 14 & Jaguarundi & 1 & 0 & 1 & 1 & 2 & 2 & 3 & 0 & 0 & 1 & 2 & 3 & 1 & 0 & 4 \\
\hline 15 & C. Chaus & 1 & 1 & 2 & 1 & 2 & 1 & 2 & 0 & 0 & 1 & 3 & 3 & 1 & 0 & 4 \\
\hline 16 & C. Dore & 1 & 0 & 1 & 1 & 1 & 1 & 2 & 0 & 0 & 1 & 3 & 3 & 1 & 0 & 4 \\
\hline 17 & C. Marguay & 2 & 0 & 1 & 1 & 1 & 1 & 2 & 0 & 0 & 1 & 3 & 3 & 1 & 0 & 4 \\
\hline 18 & C. Margerite & 1 & 1 & 1 & 1 & 1 & 1 & 2 & 0 & 0 & 1 & 2 & 3 & 0 & 0 & 4 \\
\hline 19 & C. Cafer & 3 & 0 & 1 & 1 & 1 & 1 & 2 & 0 & 0 & 1 & 3 & 3 & 1 & 1 & 4 \\
\hline 20 & C. Chine & 1 & 0 & 2 & 1 & 1 & 1 & 1 & 0 & 0 & 1 & 2 & 3 & 1 & 0 & 4 \\
\hline 21 & C. Bengale & 2 & 0 & 1 & 1 & 1 & 1 & 2 & 0 & 0 & 1 & 3 & 3 & 1 & 0 & 4 \\
\hline 22 & C. Rouilleux & 2 & 0 & 1 & 1 & 1 & 1 & 2 & 0 & 0 & 1 & 2 & 3 & 1 & 0 & 4 \\
\hline 23 & C. Malais & 1 & 1 & 1 & 1 & 1 & 1 & 1 & 0 & 0 & 1 & 3 & 3 & 1 & 0 & 4 \\
\hline 24 & C. Borneo & 1 & 0 & 1 & 1 & 1 & 1 & 2 & 0 & 0 & 1 & 3 & 3 & 1 & 0 & 4 \\
\hline 25 & C. Negripes & 2 & 0 & 1 & 1 & 1 & 1 & 1 & 0 & 0 & 1 & 2 & 3 & 1 & 1 & 4 \\
\hline 26 & C. Manul & 1 & 1 & 1 & 1 & 1 & 1 & 1 & 0 & 0 & 1 & 3 & 3 & 1 & 0 & 4 \\
\hline 27 & C. Marbre & 4 & 0 & 1 & 1 & 1 & 1 & 3 & 0 & 0 & 1 & 3 & 3 & 1 & 0 & 4 \\
\hline 28 & C. Tigrin & 2 & 0 & 1 & 1 & 1 & 1 & 2 & 0 & 0 & 1 & 3 & 3 & 1 & 0 & 4 \\
\hline 29 & C. Temminck & 1 & 0 & 1 & 1 & 1 & 1 & 2 & 0 & 0 & 1 & 3 & 3 & 1 & 0 & 4 \\
\hline 30 & C. Andes & 2 & 1 & 1 & 1 & 1 & 2 & 2 & 0 & 0 & 1 & 3 & 2 & 1 & 0 & 4 \\
\hline
\end{tabular}

\section{Appendix A3. Classification of workers}

Reference: Opitz and Schader (1984). The parameters in Table A3 are specified as follows:

1: Type of work. 2: Working speed. 3: Noise level. 4: Humidity. 5: Friendly superiors. 6: Unfriendly superiors. 7: Headman. 8: Friendly colleagues. 9: Unfriendly colleagues. 10: Colleagues. 11: Salary. 12: Fairness w.r.t. payment. 13: Career perspectives.

Table A3

Data matrix and solution classes (SC)

\begin{tabular}{rrrrrrrrrrrrrrr}
\hline & 1 & 2 & 3 & 4 & 5 & 6 & 7 & 8 & 9 & 10 & 11 & 12 & 13 & SC \\
\hline 1 & -1 & -1 & -1 & 1 & 1 & 1 & -1 & 1 & 1 & 1 & -1 & -1 & 1 & 1 \\
2 & 0 & 1 & 1 & 1 & -1 & 1 & 0 & 1 & 1 & 0 & -1 & -1 & 1 & 2 \\
3 & 1 & -1 & 1 & 1 & 1 & 1 & 1 & 1 & -1 & 0 & 1 & 1 & 1 & 3 \\
4 & 0 & -1 & 1 & 1 & 1 & 1 & 0 & 1 & -1 & 0 & -1 & -1 & -1 & 2 \\
5 & 0 & 1 & -1 & 1 & 1 & 1 & 1 & 1 & -1 & 1 & 1 & 0 & 1 & 3 \\
6 & 1 & 1 & 1 & 0 & 1 & -1 & 1 & 1 & -1 & 1 & -1 & -1 & 0 & 3 \\
7 & 1 & 1 & 1 & 1 & 1 & 1 & 1 & 1 & 1 & 0 & 0 & -1 & -1 & 3 \\
8 & 1 & 1 & 1 & 1 & 1 & -1 & 1 & 1 & 1 & 1 & 1 & -1 & 0 & 3 \\
9 & 1 & 1 & -1 & 0 & 1 & 1 & 1 & 1 & 1 & 0 & 1 & 1 & -1 & 3 \\
10 & 1 & -1 & 1 & 1 & -1 & 1 & 0 & 1 & 1 & 1 & -1 & -1 & -1 & 2 \\
11 & 0 & -1 & 1 & 1 & 1 & 1 & 1 & 1 & -1 & 0 & 0 & 0 & 0 & 3 \\
12 & 1 & -1 & 1 & -1 & 1 & 1 & 1 & -1 & 1 & 0 & 1 & -1 & 1 & 3 \\
13 & 1 & 1 & 1 & 0 & 1 & 1 & 1 & 1 & -1 & 0 & 1 & 1 & 1 & 3 \\
14 & 1 & 1 & 1 & 0 & 1 & 1 & 1 & 1 & 1 & 0 & 1 & 1 & 1 & 3 \\
15 & 0 & 1 & 1 & 1 & 1 & 1 & 1 & 1 & -1 & 0 & -1 & -1 & 0 & 3 \\
16 & 1 & 1 & 1 & 0 & 1 & 1 & 1 & 1 & -1 & 0 & 0 & 0 & 0 & 3 \\
17 & 1 & 1 & 1 & 1 & 1 & 1 & 1 & 1 & -1 & 0 & 0 & 0 & 1 & 3 \\
18 & 0 & 1 & 1 & 1 & 1 & 1 & 1 & 1 & -1 & 0 & 1 & 1 & 0 & 3 \\
19 & 0 & 1 & 1 & 1 & 1 & 1 & 1 & 1 & 1 & 0 & 1 & -1 & -1 & 3
\end{tabular}


Table A3-continued

\begin{tabular}{rrrrrrrrrrrrrrrr}
\hline & 1 & 2 & 3 & 4 & 5 & 6 & 7 & 8 & 9 & 10 & 11 & 12 & 13 & SC \\
\hline 20 & 1 & 1 & -1 & 0 & 1 & 1 & 1 & 1 & 1 & 1 & -1 & 1 & 0 & 3 \\
21 & 1 & 1 & 1 & 1 & 1 & 1 & 1 & 1 & -1 & 0 & 0 & 1 & 1 & 3 \\
22 & 1 & 1 & 1 & 0 & 1 & 1 & 1 & 1 & 1 & 1 & 1 & 1 & 1 & 3 \\
23 & 0 & -1 & 1 & 1 & 1 & 1 & 1 & 1 & 1 & 1 & 0 & -1 & 0 & 3 \\
24 & 0 & 1 & -1 & 0 & 1 & -1 & 1 & 1 & 1 & 0 & 1 & 1 & 1 & 3 \\
25 & -1 & 1 & -1 & 0 & -1 & 1 & -1 & 1 & 1 & 0 & 0 & -1 & 1 & 1 \\
26 & 0 & 1 & 1 & 1 & 1 & 1 & 0 & -1 & -1 & -1 & -1 & -1 & -1 & 2 \\
27 & 1 & 1 & 1 & 1 & -1 & 1 & 1 & 1 & 1 & 1 & 1 & 1 & 1 & 3 \\
28 & 1 & 1 & -1 & 0 & 1 & 1 & 1 & 1 & 1 & 0 & -1 & -1 & 1 & 3 \\
29 & 1 & 1 & -1 & 1 & 1 & 1 & 1 & 1 & 1 & 0 & 1 & 1 & 1 & 3 \\
30 & 0 & 1 & -1 & 0 & 1 & 1 & -1 & 1 & 1 & 0 & 1 & 1 & 1 & 3 \\
31 & -1 & -1 & 1 & 1 & -1 & 1 & 1 & -1 & -1 & 1 & 0 & -1 & -1 & 2 \\
32 & 0 & -1 & 1 & 1 & -1 & -1 & -1 & 1 & 1 & 0 & -1 & -1 & 0 & 2 \\
33 & 0 & -1 & 1 & 1 & 1 & 1 & 1 & 1 & 1 & 0 & 1 & 1 & 1 & 3 \\
34 & 0 & 1 & 1 & 1 & -1 & -1 & 0 & 1 & -1 & 0 & 0 & 1 & 1 & 2 \\
\hline
\end{tabular}

(1): Satisfied/many $(5,6,8,9),(-1)$ : Unsatisfied/few, (0): Undecided.

\section{Appendix A4. Classification of cars}

Reference: Hartigan (1975). The parameters in Table A4 are specified as follows:

1: Brake system. 2: Fuel system. 3: Electrical 4: Exhaust. 5: Steering. 6: Engine, mechanical. 7: Rattles and squeaks. 8: Rear axle. 9: Rust. 10: Shock absorbers. 11: Transmission, clutch. 12: Wheel alignment. 13: Other.

Table A4

Data matrix and solution classes (SC)

\begin{tabular}{llllllllllllllll}
\hline \multicolumn{1}{l}{ Car } & 1 & 2 & 3 & 4 & 5 & 6 & 7 & 8 & 9 & 10 & 11 & 12 & 13 & SC \\
\hline 1 & AMC Ambassador 8 & 1 & 0 & 0 & 0 & 0 & 0 & 0 & 1 & 0 & 0 & 0 & 0 & 0 & 1 \\
2 & Buick Special 6 & 0 & 0 & 0 & 0 & 0 & 0 & 1 & 0 & 1 & 0 & 0 & 0 & 1 & 1 \\
3 & Buick Special 8 & 0 & 0 & 0 & 0 & 0 & 0 & 1 & 0 & 0 & 1 & 0 & 1 & 1 & 1 \\
4 & Buick 8 Full & 0 & 0 & 0 & 1 & 0 & 1 & 1 & 0 & 1 & 1 & 0 & 1 & 0 & 1 \\
5 & Buick Riviera & 0 & 0 & 1 & 1 & 0 & 0 & 0 & 0 & 0 & 1 & 0 & 0 & 0 & 1 \\
6 & Cadillac Chevy II & 0 & 1 & 0 & 0 & 1 & 0 & 1 & 1 & 1 & 0 & 0 & 1 & 0 & 2 \\
7 & Chevelle 6 & 0 & 0 & 0 & 0 & 0 & 1 & 1 & 0 & 1 & 0 & 0 & 0 & 0 & 1 \\
8 & Chevelle 8 & 0 & 1 & 0 & 1 & 1 & 0 & 1 & 0 & 1 & 1 & 0 & 1 & 0 & 2 \\
9 & Chevrolet Full & 0 & 1 & 1 & 1 & 1 & 0 & 1 & 1 & 1 & 1 & 1 & 1 & 0 & 2 \\
10 & Corvair 6 & 0 & 1 & 0 & 0 & 1 & 1 & 0 & 1 & 0 & 1 & 1 & 1 & 1 & 3 \\
11 & Corvette & 0 & 0 & 0 & 1 & 0 & 0 & 1 & 1 & 0 & 0 & 1 & 0 & 0 & 1 \\
12 & Chrysler Newport & 1 & 0 & 0 & 0 & 0 & 0 & 0 & 0 & 0 & 0 & 0 & 0 & 0 & 1 \\
13 & New Yorker & 1 & 0 & 0 & 0 & 0 & 0 & 0 & 1 & 0 & 0 & 0 & 0 & 1 & 1 \\
14 & Dodge Full Size & 1 & 0 & 0 & 0 & 0 & 0 & 1 & 0 & 0 & 1 & 0 & 0 & 0 & 1 \\
15 & Falcon 6 & 0 & 0 & 0 & 0 & 0 & 0 & 1 & 0 & 0 & 0 & 1 & 1 & 0 & 1 \\
16 & Fairlane 6 & 0 & 0 & 0 & 0 & 0 & 0 & 1 & 0 & 0 & 0 & 0 & 0 & 0 & 1 \\
17 & Fairlane 8 & 0 & 0 & 0 & 1 & 0 & 0 & 1 & 1 & 0 & 0 & 0 & 1 & 0 & 1 \\
18 & Ford, Full Size & 0 & 0 & 0 & 1 & 1 & 0 & 0 & 0 & 0 & 1 & 0 & 1 & 1 & 1 \\
19 & Thunderbird & 0 & 0 & 1 & 0 & 1 & 1 & 0 & 0 & 0 & 0 & 0 & 1 & 1 & 1
\end{tabular}


Table A4-continued

\begin{tabular}{|c|c|c|c|c|c|c|c|c|c|c|c|c|c|c|c|}
\hline & Car & 1 & 2 & 3 & 4 & 5 & 6 & 7 & 8 & 9 & 10 & 11 & 12 & 13 & $\mathrm{SC}$ \\
\hline 20 & Mercury Full & 0 & 0 & 0 & 0 & 0 & 0 & 0 & 0 & 0 & 0 & 0 & 1 & 0 & 1 \\
\hline 21 & Olds Full & 1 & 1 & 0 & 0 & 0 & 0 & 1 & 0 & 0 & 1 & 0 & 1 & 0 & 1 \\
\hline 22 & Plymouth Full & 1 & 0 & 0 & 0 & 0 & 0 & 0 & 0 & 0 & 0 & 0 & 0 & 0 & 1 \\
\hline 23 & Pontiac Tempest & 0 & 1 & 0 & 0 & 0 & 0 & 1 & 0 & 1 & 1 & 0 & 1 & 0 & 1 \\
\hline 24 & Pontiac Full & 1 & 1 & 1 & 0 & 0 & 0 & 1 & 0 & 1 & 1 & 0 & 1 & 0 & 2 \\
\hline 25 & Rambler Rebel 6 & 0 & 0 & 1 & 0 & 0 & 0 & 0 & 1 & 0 & 0 & 1 & 0 & 1 & 1 \\
\hline 26 & Mercedes & 0 & 0 & 0 & 0 & 0 & 0 & 0 & 0 & 0 & 1 & 0 & 0 & 0 & 1 \\
\hline 27 & MG 1100 & 0 & 0 & 1 & 1 & 0 & 0 & 0 & 0 & 0 & 0 & 0 & 0 & 0 & 1 \\
\hline 28 & Peugeot & 0 & 0 & 0 & 0 & 0 & 0 & 0 & 0 & 0 & 0 & 1 & 0 & 0 & 1 \\
\hline 29 & Porsche & 0 & 0 & 0 & 0 & 0 & 0 & 0 & 0 & 0 & 0 & 0 & 0 & 0 & 1 \\
\hline 30 & Renault & 0 & 0 & 0 & 0 & 0 & 0 & 0 & 0 & 0 & 0 & 1 & 0 & 0 & 1 \\
\hline 31 & Volvo & 0 & 0 & 0 & 1 & 0 & 0 & 0 & 1 & 0 & 0 & 0 & 0 & 0 & 1 \\
\hline 32 & VW bug & 1 & 0 & 1 & 1 & 1 & 1 & 0 & 0 & 0 & 0 & 1 & 0 & 0 & 4 \\
\hline 33 & VW bus & 0 & 0 & 1 & 0 & 0 & 1 & 0 & 0 & 1 & 0 & 1 & 0 & 0 & 1 \\
\hline
\end{tabular}

The " 1 " means greater than average frequency of repair in 1962-1967.

\section{Appendix A5. Classification of micro computers}

Reference: Chah (1984). The parameters in Table A5 are specified as follows:

1: Color Monitor. 2: Disk Operating System CP/M.3: Disk Operating System MS-DOS. 4: Disk Operating System "other". 5: Processor (1: 8 bits, 2: 16 bits, 3: 32 bits). 6: Parallel Interface. 7: Serial Interface. 8: IEEE 488 Interface. 9: Hard Disk (0: if it does not exist, 1: $5 \mathrm{Mb}, 2: 10 \mathrm{Mb}$ ). 10: Number of diskette drives (1 or 2). 11: Price (F.F.). 12: Random Access Memory, configuration (Kb). 13: Random Access Memory, maximum (Kb). 14: Mass Storage, Diskette Unit (Kb).

Table A5

Data matrix and solution classes (SC)

\begin{tabular}{|c|c|c|c|c|c|c|c|c|c|c|c|c|c|c|c|c|}
\hline & Computer & 1 & 2 & 3 & 4 & 5 & 6 & 7 & 8 & 9 & 10 & 11 & 12 & 13 & 14 & $\mathrm{SC}$ \\
\hline 1 & PAP & 0 & 0 & 2 & 0 & 2 & 1 & 1 & 1 & 0 & 1 & 20000 & 192 & 512 & 720 & 1 \\
\hline 2 & QX 10 & 1 & 2 & 0 & 0 & 1 & 2 & 1 & 1 & 0 & 2 & 23500 & 192 & 250 & 320 & 2 \\
\hline 3 & MACINTOSH & 0 & 0 & 0 & 2 & 3 & 0 & 2 & 0 & 0 & 1 & 26000 & 128 & 512 & 400 & 3 \\
\hline 4 & TI PC & 2 & 2 & 2 & 0 & 2 & 2 & 1 & 0 & 0 & 1 & 26300 & 128 & 768 & 320 & 4 \\
\hline 5 & PAP (2) & 0 & 0 & 2 & 0 & 2 & 1 & 1 & 1 & 0 & 2 & 27200 & 192 & 512 & 720 & 1 \\
\hline 6 & APRICOT & 0 & 0 & 2 & 0 & 2 & 2 & 2 & 1 & 0 & 2 & 28400 & 256 & 768 & 315 & 4 \\
\hline 7 & Z 150 & 0 & 0 & 2 & 0 & 2 & 2 & 2 & 0 & 0 & 2 & 28500 & 320 & 640 & 360 & 4 \\
\hline 8 & GOUPIL 3 & 0 & 2 & 0 & 0 & 1 & 2 & 2 & 0 & 0 & 2 & 29700 & 64 & 1024 & 360 & 2 \\
\hline 9 & APPLE 3 & 0 & 0 & 0 & 2 & 1 & 1 & 2 & 1 & 1 & 1 & 35000 & 256 & 256 & 140 & 5 \\
\hline 10 & TANDY 2000 & 1 & 0 & 2 & 0 & 2 & 2 & 2 & 0 & 0 & 2 & 30200 & 128 & 768 & 720 & 4 \\
\hline 11 & IBM PC & 1 & 1 & 2 & 0 & 2 & 2 & 1 & 0 & 0 & 2 & 36100 & 128 & 640 & 320 & 4 \\
\hline 12 & TI PC (2) & 2 & 2 & 2 & 0 & 2 & 2 & 1 & 0 & 0 & 2 & 39000 & 256 & 768 & 320 & 4 \\
\hline 13 & APPLE $2 \mathrm{E}$ & 1 & 1 & 0 & 2 & 1 & 1 & 1 & 1 & 1 & 1 & 39400 & 128 & 832 & 140 & 5 \\
\hline 14 & TELE PC & 1 & 0 & 2 & 0 & 2 & 2 & 2 & 1 & 2 & 1 & 59200 & 256 & 640 & 360 & 4 \\
\hline 15 & PAP (3) & 0 & 0 & 2 & 0 & 2 & 1 & 1 & 1 & 2 & 1 & 47400 & 192 & 512 & 720 & 1 \\
\hline 16 & IBM PC XT & 1 & 1 & 2 & 0 & 2 & 2 & 1 & 0 & 2 & 1 & 51000 & 128 & 640 & 320 & 4 \\
\hline 17 & Z $150(2)$ & 0 & 2 & 2 & 0 & 2 & 2 & 2 & 0 & 2 & 2 & 51500 & 320 & 640 & 360 & 4 \\
\hline 18 & TANDY $2000(2)$ & 1 & 0 & 2 & 0 & 2 & 2 & 2 & 0 & 2 & 2 & 52200 & 128 & 768 & 720 & 4 \\
\hline
\end{tabular}


Table A5--continued

\begin{tabular}{|c|c|c|c|c|c|c|c|c|c|c|c|c|c|c|c|c|}
\hline & Computer & 1 & 2 & 3 & 4 & 5 & 6 & 7 & 8 & 9 & 10 & 11 & 12 & 13 & 14 & $\mathrm{SC}$ \\
\hline 19 & VICTOR S1 & 1 & 2 & 2 & 0 & 2 & 2 & 2 & 1 & 2 & 2 & 66000 & 256 & 896 & 1228 & 4 \\
\hline 20 & T 200 & 0 & 2 & 0 & 0 & 1 & 2 & 2 & 0 & 0 & 2 & 22500 & 64 & 64 & 256 & 2 \\
\hline 21 & AS 100 & 2 & 1 & 2 & 0 & 2 & 2 & 2 & 2 & 0 & 2 & 32000 & 128 & 512 & 640 & 4 \\
\hline 22 & MZ 35 & 1 & 0 & 2 & 2 & 1 & 2 & 2 & 0 & 0 & 2 & 34000 & 136 & 372 & 400 & 4 \\
\hline 23 & BASIS 108 & 1 & 0 & 0 & 2 & 1 & 2 & 2 & 1 & 0 & 2 & 28500 & 384 & 384 & 160 & 3 \\
\hline 24 & LISA 2 & 0 & 0 & 0 & 2 & 3 & 2 & 1 & 1 & 0 & 1 & 35500 & 512 & 512 & 400 & 3 \\
\hline 25 & EUROPE PC & 1 & 0 & 2 & 0 & 2 & 2 & 2 & 0 & 2 & 1 & 47400 & 128 & 1024 & 327 & 4 \\
\hline 26 & PSI 80 & 0 & 2 & 0 & 0 & 1 & 2 & 2 & 0 & 0 & 2 & 47800 & 80 & 256 & 308 & 2 \\
\hline 27 & CORONA PC 2 & 1 & 1 & 2 & 0 & 2 & 2 & 2 & 1 & 2 & 2 & 45000 & 256 & 512 & 320 & 4 \\
\hline 28 & OPLITE & 1 & 0 & 2 & 0 & 2 & 2 & 2 & 0 & 0 & 2 & 33500 & 256 & 640 & 360 & 4 \\
\hline 29 & HORIZON & 0 & 2 & 0 & 0 & 1 & 2 & 2 & 0 & 0 & 2 & 35000 & 64 & 576 & 360 & 2 \\
\hline 30 & FOXY & 1 & 1 & 2 & 0 & 2 & 2 & 2 & 1 & 2 & 1 & 51000 & 256 & 1024 & 360 & 4 \\
\hline 31 & SKS 2500 & 0 & 2 & 0 & 0 & 1 & 1 & 2 & 1 & 0 & 2 & 32000 & 64 & 256 & 800 & 2 \\
\hline 32 & ZEPHYR & 0 & 2 & 0 & 0 & 1 & 2 & 2 & 0 & 0 & 2 & 41400 & 64 & 64 & 640 & 2 \\
\hline 33 & MBC 4050 & 0 & 2 & 0 & 0 & 2 & 2 & 2 & 0 & 0 & 2 & 35600 & 256 & 1024 & 640 & 2 \\
\hline 34 & SANCO 8000 & 0 & 2 & 0 & 0 & 1 & 2 & 2 & 0 & 0 & 2 & 26100 & 70 & 192 & 400 & 2 \\
\hline 35 & IPC MODEL 15 & 0 & 2 & 0 & 0 & 1 & 0 & 2 & 0 & 0 & 2 & 43000 & 64 & 512 & 782 & 2 \\
\hline 36 & DESKTOP 10 & 0 & 1 & 2 & 0 & 2 & 0 & 2 & 1 & 0 & 2 & 44800 & 128 & 768 & 360 & 4 \\
\hline 37 & LISA 2-S & 0 & 0 & 0 & 2 & 3 & 2 & 1 & 1 & 1 & 1 & 47400 & 512 & 1024 & 400 & 3 \\
\hline 38 & NEC PC 8000 & 0 & 0 & 0 & 2 & 1 & 2 & 1 & 1 & 0 & 2 & 31800 & 32 & 64 & 320 & 3 \\
\hline 39 & M 20 & 0 & 0 & 0 & 2 & 2 & 2 & 2 & 1 & 0 & 2 & 21600 & 128 & 512 & 286 & 3 \\
\hline 40 & TRS 80 MOD 12 & 0 & 0 & 0 & 2 & 1 & 2 & 2 & 0 & 0 & 1 & 32000 & 80 & 768 & 422 & 2 \\
\hline
\end{tabular}

(0): The feature does not exist, (1): The feature is optional, (2): The feature exists.

\section{Appendix A6. Votes of 54 member states of the UNO}

Reference: Marcotorchino (1981). See Table A6.

\section{Table A6}

Data matrix and solution classes (SC)

\begin{tabular}{|c|c|c|c|c|c|c|c|c|c|c|c|}
\hline & States & $a b c$ & $\mathrm{SC}$ & & States & $\mathrm{a} b \mathrm{c}$ & SC & & States & $a b c$ & $\mathrm{SC}$ \\
\hline 1 & U.S.A. & $\begin{array}{lll}33 & 1\end{array}$ & 1 & 16 & COLU & 521 & 3 & 31 & LUXE & 331 & 1 \\
\hline 2 & CANA & 321 & 1 & 17 & VENE & $\begin{array}{lll}3 & 1 & 1\end{array}$ & 1 & 32 & FRAN & 323 & 4 \\
\hline 3 & CUBA & 153 & 2 & 18 & GUYA & $\begin{array}{lll}5 & 1 & 1\end{array}$ & 3 & 33 & SPAI & 321 & 1 \\
\hline 4 & HAIT & $\begin{array}{lll}5 & 1 & 1\end{array}$ & 3 & 19 & ECUA & 352 & 5 & 34 & PORT & 532 & 6 \\
\hline 5 & DOMI & 111 & 2 & 20 & PERU & $\begin{array}{lll}3 & 1 & 1\end{array}$ & 1 & 35 & POLA & 113 & 2 \\
\hline 6 & JAMA & $\begin{array}{lll}3 & 1 & 1\end{array}$ & 1 & 21 & BRAZ & 321 & 1 & 36 & AUST & 322 & 4 \\
\hline 7 & TRIN & $\begin{array}{lll}3 & 1 & 1\end{array}$ & 1 & 22 & BOLI & 511 & 3 & 37 & HUNG & 113 & 2 \\
\hline 8 & BARB & $\begin{array}{lll}5 & 12\end{array}$ & 3 & 23 & PARA & $\begin{array}{lll}32 & 1\end{array}$ & 1 & 38 & CZEC & $\begin{array}{lll}113 & 1\end{array}$ & 2 \\
\hline 9 & MEXI & $\begin{array}{lll}3 & 1 & 1\end{array}$ & 1 & 24 & CHIL & $\begin{array}{lll}3 & 1 & 1\end{array}$ & 1 & 39 & ITAL & 321 & 1 \\
\hline 10 & GUAT & $\begin{array}{lll}31 & 1\end{array}$ & 1 & 25 & ARGE & $\begin{array}{lll}3 & 1 & 1\end{array}$ & 1 & 40 & MALT & 551 & 3 \\
\hline 11 & HOND & 321 & 1 & 26 & URUG & 351 & 1 & 41 & ALBA & 153 & 2 \\
\hline 12 & EL $S$ & $\begin{array}{lll}3 & 2 & 1\end{array}$ & 1 & 27 & U.K. & 331 & 1 & 42 & YUGO & 113 & 2 \\
\hline 13 & NICA & $\begin{array}{lll}3 & 2 & 1\end{array}$ & 1 & 28 & IREL & 321 & 1 & 43 & GREE & $\begin{array}{lll}3 & 1 & 1\end{array}$ & 1 \\
\hline 14 & COST & $\begin{array}{lll}3 & 1 & 1\end{array}$ & 1 & 29 & NETH & 331 & 1 & 44 & CYPR & $\begin{array}{lll}3 & 1 & 1\end{array}$ & 1 \\
\hline 15 & PANA & $\begin{array}{lll}3 & 1 & 1\end{array}$ & 1 & 30 & BELG & 331 & 1 & 45 & BULG & $\begin{array}{lll}1 & 1 & 3\end{array}$ & 2 \\
\hline
\end{tabular}


Table A6-continued

\begin{tabular}{|c|c|c|c|c|c|c|c|c|c|c|c|}
\hline & States & $\mathrm{a} b \mathrm{~b}$ & $\mathrm{SC}$ & & States & $a b c$ & $\mathrm{SC}$ & & States & $\mathrm{a} b \mathrm{c}$ & SC \\
\hline 46 & ROMA & 113 & 2 & 49 & BYEL & 113 & 2 & 52 & NORW & 323 & 4 \\
\hline 47 & USSR & 113 & 2 & 50 & FINL & 223 & 4 & 53 & DENM & 323 & 4 \\
\hline 48 & UKRA & 113 & 2 & 51 & SWED & 323 & 4 & 54 & ICEL & 321 & 1 \\
\hline
\end{tabular}

(1): In favour, (2): Against, (3): Abstaining, (5): Absent.

\section{Appendix A7. Votes of all member states of the UNO}

Reference: UNO (1985).

Remark. (a) The votes considered here are those which were recorded when the session was held.

(b) The states which announced that they were not participating in a vote are considered here as "absent".

\section{A7.1. Votes on Resolutions 39/119-120-121}

The parameters in Table A7.1 are specified as follows:
A: 39/119-Situation of human rights and fundamental freedoms in El Salvador.
B: $39 / 120-$ Situation of human rights and fundamental freedoms in Guatemala.
C: 39/121-Situation of human rights and fundamental freedoms in Chile.

The solution classes in Table A7.1 are specified as follows:

SC1: Solution classes with all states.

SC2: Solution classes without considering states which were absent at least once.

Table A7.1

Data matrix and solution classes

\begin{tabular}{|c|c|c|c|c|c|c|}
\hline & States & A & $\mathrm{B}$ & $\mathrm{C}$ & SC1 & $\mathrm{SC} 2$ \\
\hline 1 & Afganistan & 0 & 0 & 0 & 1 & 1 \\
\hline 2 & Albania & 0 & 3 & 3 & 2 & \\
\hline 3 & Algeria & 0 & 0 & 0 & 1 & 1 \\
\hline 4 & Angola & 0 & 0 & 0 & 1 & 1 \\
\hline 5 & Antigua and Barbuda & 3 & 3 & 3 & 2 & \\
\hline 6 & Argentina & 0 & 0 & 0 & 1 & 1 \\
\hline 7 & Australia & 0 & 0 & 0 & 1 & 1 \\
\hline 8 & Austria & 0 & 0 & 0 & 1 & 1 \\
\hline 9 & Bahamas & 2 & 2 & 2 & 3 & 2 \\
\hline 10 & Bahrain & 0 & 0 & 0 & 1 & 1 \\
\hline 11 & Bangladesh & 1 & 1 & 1 & 4 & 3 \\
\hline 12 & Barbados & 0 & 0 & 0 & 1 & 1 \\
\hline 13 & Belgium & 0 & 0 & 0 & 1 & 1 \\
\hline 14 & Belize & 2 & 2 & 2 & 3 & 2 \\
\hline 15 & Benin & 0 & 0 & 0 & 1 & 1 \\
\hline 16 & Bhutan & 2 & 2 & 2 & 3 & 2 \\
\hline 17 & Bolivia & 3 & 3 & 3 & 2 & \\
\hline 18 & Botswana & 0 & 0 & 0 & 1 & 1 \\
\hline 19 & Brazil & 2 & 2 & 1 & 3 & 2 \\
\hline 20 & Brunei Darussalam & 2 & 2 & 2 & 3 & 2 \\
\hline 21 & Bulgaria & 0 & 0 & 0 & 1 & 1 \\
\hline 22 & Burkina Faso & 0 & 0 & 0 & 1 & 1 \\
\hline 23 & Burma & 2 & 2 & 2 & 3 & 2 \\
\hline
\end{tabular}


Table A7.1—continued

\begin{tabular}{|c|c|c|c|c|c|c|}
\hline & States & A & B & $\mathrm{C}$ & $\mathrm{SC} 1$ & $\mathrm{SC} 2$ \\
\hline 24 & Burundi & 0 & 0 & 0 & 1 & 1 \\
\hline 25 & Byelorussia & 0 & 0 & 0 & 1 & 1 \\
\hline 26 & Cameroon & 3 & 3 & 2 & 2 & \\
\hline 27 & Canada & 0 & 0 & 0 & 1 & 1 \\
\hline 28 & Cape Verde & 0 & 0 & 0 & 1 & 1 \\
\hline 29 & Central African Republic & 2 & 2 & 2 & 3 & 2 \\
\hline 30 & Chad & 2 & 2 & 2 & 3 & 2 \\
\hline 31 & Chile & 1 & 1 & 1 & 4 & 3 \\
\hline 32 & China & 2 & 2 & 2 & 3 & 2 \\
\hline 33 & Colombia & 0 & 2 & 3 & 5 & \\
\hline 34 & Comoros & 3 & 3 & 3 & 2 & \\
\hline 35 & Congo & 0 & 0 & 0 & 1 & 1 \\
\hline 36 & Costa Rica & 0 & 2 & 0 & 1 & 1 \\
\hline 37 & Cuba & 0 & 0 & 0 & 1 & 1 \\
\hline 38 & Cyprus & 0 & 0 & 0 & 1 & 1 \\
\hline 39 & Czechoslovakia & 0 & 0 & 0 & 1 & 1 \\
\hline 40 & Democratic Kampuchea & 2 & 2 & 2 & 3 & 2 \\
\hline 41 & Democratic Yemen & 0 & 0 & 0 & 1 & 1 \\
\hline 42 & Denmark & 0 & 0 & 0 & 1 & 1 \\
\hline 43 & Djibouti & 3 & 3 & 3 & 2 & \\
\hline 44 & Dominica & 3 & 3 & 3 & 2 & \\
\hline 45 & Dominican Republic & 0 & 2 & 0 & 1 & 1 \\
\hline 46 & Ecuador & 2 & 2 & 2 & 3 & 2 \\
\hline 47 & Egypt & 0 & 2 & 2 & 3 & 2 \\
\hline 48 & El Salvador & 1 & 1 & 1 & 4 & 3 \\
\hline 49 & Equatorial Guinea & 2 & 2 & 0 & 3 & 2 \\
\hline 50 & Ethiopia & 0 & 0 & 0 & 1 & 1 \\
\hline 51 & Federal Republic of Germany & 2 & 0 & 0 & 1 & 1 \\
\hline 52 & Fiji & 2 & 2 & 2 & 3 & 2 \\
\hline 53 & Finland & 0 & 0 & 0 & 1 & 1 \\
\hline 54 & France & 0 & 0 & 0 & 1 & 1 \\
\hline 55 & Gabon & 2 & 2 & 2 & 3 & 2 \\
\hline 56 & Gambia & 0 & 0 & 0 & 1 & 1 \\
\hline 57 & German Democratic Republic & 0 & 0 & 0 & 1 & 1 \\
\hline 58 & Ghana & 0 & 0 & 0 & 1 & 1 \\
\hline 59 & Greece & 0 & 0 & 0 & 1 & 1 \\
\hline 60 & Grenada & 3 & 3 & 3 & 2 & \\
\hline 61 & Guatemala & 1 & 1 & 1 & 4 & 3 \\
\hline 62 & Guinea & 0 & 2 & 0 & 1 & 1 \\
\hline 63 & Guinea-Bissau & 3 & 3 & 3 & 2 & \\
\hline 64 & Guyana & 0 & 0 & 0 & 1 & 1 \\
\hline 65 & Haiti & 1 & 1 & 1 & 4 & 3 \\
\hline 66 & Honduras & 1 & 2 & 2 & 3 & 2 \\
\hline 67 & Hungary & 0 & 0 & 0 & 1 & 1 \\
\hline 68 & Iceland & 0 & 0 & 0 & 1 & 1 \\
\hline 69 & India & 0 & 0 & 0 & 1 & 1 \\
\hline 70 & Indonesia & 1 & 1 & 1 & 4 & 3 \\
\hline 71 & Iran & 0 & 0 & 0 & 1 & 1 \\
\hline 72 & Iraq & 0 & 0 & 3 & 1 & \\
\hline 73 & Ireland & 0 & 0 & 0 & 1 & 1 \\
\hline 74 & Israel & 3 & 3 & 3 & 2 & \\
\hline
\end{tabular}


Table A7.1-continued

\begin{tabular}{|c|c|c|c|c|c|c|}
\hline & States & A & $\mathbf{B}$ & $\mathrm{C}$ & $\mathrm{SC} 1$ & $\mathrm{SC} 2$ \\
\hline 75 & Italy & 0 & 0 & 0 & 1 & 1 \\
\hline 76 & Ivory Coast & 2 & 2 & 2 & 3 & 2 \\
\hline 77 & Jamaica: & 0 & 0 & 0 & 1 & 1 \\
\hline 78 & Japan & 2 & 2 & 2 & 3 & 2 \\
\hline 79 & Jordan & 2 & 2 & 2 & 3 & 2 \\
\hline 80 & Kenya & 0 & 0 & 0 & 1 & 1 \\
\hline 81 & Kuwait & 0 & 0 & 0 & 1 & 1 \\
\hline 82 & Lao People's Democratic Rep. & 0 & 0 & 0 & 1 & 1 \\
\hline 83 & Lebanon & 3 & 3 & 1 & 2 & \\
\hline 84 & Lesotho & 0 & 0 & 0 & 1 & 1 \\
\hline 85 & Liberia & 2 & 2 & 2 & 3 & 2 \\
\hline 86 & Libya & 0 & 0 & 0 & 1 & 1 \\
\hline 87 & Luxembourg & 0 & 0 & 0 & 1 & 1 \\
\hline 88 & Madagascar & 0 & 0 & 0 & 1 & 1 \\
\hline 89 & Malawi & 2 & 2 & 2 & 3 & 2 \\
\hline 90 & Malaysia & 2 & 2 & 2 & 3 & 2 \\
\hline 91 & Maldives & 2 & 2 & 0 & 3 & 2 \\
\hline 92 & Mali & 0 & 0 & 0 & 1 & 1 \\
\hline 93 & Malta & 0 & 0 & 0 & 1 & 1 \\
\hline 94 & Mauritania & 0 & 0 & 0 & 1 & 1 \\
\hline 95 & Mauritius & 0 & 0 & 0 & 1 & 1 \\
\hline 96 & Mexico & 0 & 0 & 0 & 1 & 1 \\
\hline 97 & Mongolia & 0 & 0 & 0 & 1 & 1 \\
\hline 98 & Morocco & 1 & 1 & 1 & 4 & 3 \\
\hline 99 & Mozambique & 0 & 0 & 0 & 1 & 1 \\
\hline 100 & Nepal & 2 & 2 & 2 & 3 & 2 \\
\hline 101 & Netherlands & 0 & 0 & 0 & 1 & 1 \\
\hline 102 & New Zealand & 0 & 0 & 0 & 1 & 1 \\
\hline 103 & Nicaragua & 0 & 0 & 0 & 1 & 1 \\
\hline 104 & Niger & 2 & 2 & 2 & 3 & 2 \\
\hline 105 & Nigeria & 0 & 2 & 2 & 3 & 2 \\
\hline 106 & Norway & 0 & 0 & 0 & 1 & 1 \\
\hline 107 & Oman & 2 & 2 & 2 & 3 & 2 \\
\hline 108 & Pakistan & 2 & 1 & 1 & 4 & 3 \\
\hline 109 & Panama & 0 & 2 & 2 & 3 & 2 \\
\hline 110 & Papua New Guinea & 2 & 2 & 2 & 3 & 2 \\
\hline 111 & Paraguay & 1 & 1 & 1 & 4 & 3 \\
\hline 112 & Peru & 0 & 2 & 2 & 3 & 2 \\
\hline 113 & Philippines & 2 & 2 & 2 & 3 & 2 \\
\hline 114 & Poland & 0 & 0 & 0 & 1 & 1 \\
\hline 115 & Portugal & 0 & 0 & 0 & 1 & 1 \\
\hline 116 & Qatar & 0 & 0 & 0 & 1 & 1 \\
\hline 117 & Romania & 2 & 2 & 0 & 3 & 2 \\
\hline 118 & Rwanda & 0 & 0 & 0 & 1 & 1 \\
\hline 119 & Saint Lucia & 3 & 3 & 3 & 2 & \\
\hline 120 & Saint Vicent & 0 & 3 & 2 & 6 & \\
\hline 121 & Samoa & 0 & 0 & 0 & 1 & 1 \\
\hline 122 & Sao Tome and Principe & 0 & 0 & 0 & 1 & 1 \\
\hline 123 & Saudi Arabia & 0 & 0 & 2 & 1 & 1 \\
\hline 124 & Senegal & 0 & 0 & 0 & 1 & 1 \\
\hline 125 & Seychelles & 0 & 0 & 0 & 1 & 1 \\
\hline
\end{tabular}


Table A7.1-continued

\begin{tabular}{|c|c|c|c|c|c|c|}
\hline & States & A & $\mathrm{B}$ & $\mathrm{C}$ & $\mathrm{SC} 1$ & $\mathrm{SC} 2$ \\
\hline 126 & Sierra Leone & 0 & 0 & 0 & 1 & 1 \\
\hline 127 & Singapore & 2 & 2 & 2 & 3 & 2 \\
\hline 128 & Solomon Islands & 3 & 3 & 3 & 2 & \\
\hline 129 & Somalia & 2 & 2 & 2 & 3 & 2 \\
\hline 130 & Spain & 0 & 0 & 0 & 1 & 1 \\
\hline 131 & Sri Lanka & 2 & 2 & 0 & 3 & 2 \\
\hline 132 & St. Christopher and Nevis & 3 & 3 & 3 & 2 & \\
\hline 133 & Sudan & 2 & 2 & 2 & 3 & 2 \\
\hline 134 & Suriname & 2 & 2 & 2 & 3 & 2 \\
\hline 135 & Swaziland & 0 & 0 & 0 & 1 & 1 \\
\hline 136 & Sweden & 0 & 0 & 0 & 1 & 1 \\
\hline 137 & Syria & 0 & 0 & 3 & 1 & \\
\hline 138 & Thailand & 2 & 2 & 2 & 3 & 2 \\
\hline 139 & Togo & 0 & 0 & 0 & 1 & 1 \\
\hline 140 & Trinidad Tobago & 2 & 2 & 2 & 3 & 2 \\
\hline 141 & Tunisia & 0 & 0 & 0 & 1 & 1 \\
\hline 142 & Turkey & 2 & 2 & 2 & 3 & 2 \\
\hline 143 & Uganda & 0 & 0 & 0 & 1 & 1 \\
\hline 144 & Ukraine & 0 & 0 & 0 & 1 & 1 \\
\hline 145 & USSR & 0 & 0 & 0 & 1 & 1 \\
\hline 146 & United Arab Emirates & 0 & 0 & 0 & 1 & 1 \\
\hline 147 & United Kingdom & 2 & 0 & 0 & 1 & 1 \\
\hline 148 & United Republic of Tanzania & 0 & 0 & 0 & 1 & 1 \\
\hline 149 & United States of America & 1 & 1 & 1 & 4 & 3 \\
\hline 150 & Uruguay & 1 & 1 & 1 & 4 & 3 \\
\hline 151 & Vanuatu & 0 & 0 & 0 & 1 & 1 \\
\hline 152 & Venezuela & 0 & 2 & 0 & 1 & 1 \\
\hline 153 & Viet Nam & 0 & 0 & 0 & 1 & 1 \\
\hline 154 & Yemen & 2 & 2 & 2 & 3 & 2 \\
\hline 155 & Yugoslavia & 0 & 0 & 0 & 1 & 1 \\
\hline 156 & Zaire & 2 & 2 & 2 & 3 & 2 \\
\hline 157 & Zambia & 0 & 0 & 0 & 1 & 1 \\
\hline 158 & Zimbabwe & 3 & 0 & 0 & 1 & \\
\hline
\end{tabular}

A7.2. Votes on Resolution 39/148: "Review of the implementation of the recomendations and decisions adopted by the General Assembly at its tenth special session" The parameters in Table A7.2 are specified as follows:

A: Unilateral nuclear disarmament measures. B: Bilateral nuclear arms negotiations. C: Nuclear weapons in all aspects. D: Non-use of nuclear weapons and prevention of nuclear war. E: Prohibition of the nuclear neutron weapon. F: Climatic effects of nuclear war: nuclear winter. G: Bilateral nuclear-arms negotiations. H: United Nations Institute for Disarmament Research. J: Disarmament Week. K: Cessation of the nuclear-arms race and nuclear disarmament. L: Implementation of the recomendations and decisions of the tenth special session. $\mathrm{M}$ : International co-operation for disarmament. N: Report of the Conference on Disarmament. O: Implementation of the recomendations and decisions of the tenth special session. P: Prevention of nuclear war.

The solution classes in Table A7.2 are specified as follows:

SC1: Solution class considering all states.

SC2: Solution class without considering states which were absent at least 8 times. 
Table A7.2

Data matrix and solution classes

\begin{tabular}{|c|c|c|c|c|c|c|c|c|c|c|c|c|c|c|c|c|c|}
\hline$(*)$ & A & B & $\mathrm{C}$ & $\mathrm{D}$ & $\mathrm{E}$ & $\mathrm{F}$ & $G$ & $\mathrm{H}$ & $\mathrm{J}$ & K & $\mathrm{L}$ & $\mathbf{M}$ & $\mathrm{N}$ & 0 & $\mathrm{P}$ & SC1 & $\mathrm{SC} 2$ \\
\hline 1 & 0 & 1 & 0 & 0 & 0 & 0 & 0 & 0 & 0 & 0 & 0 & 0 & 0 & 0 & 0 & 1 & 1 \\
\hline 2 & 3 & 3 & 3 & 3 & 3 & 3 & 3 & 3 & 3 & 3 & 3 & 3 & 3 & 3 & 3 & 2 & \\
\hline 3 & 0 & 2 & 0 & 0 & 0 & 0 & 0 & 0 & 0 & 0 & 0 & 0 & 0 & 0 & 0 & 1 & 1 \\
\hline 4 & 0 & 1 & 0 & 0 & 0 & 0 & 0 & 0 & 0 & 0 & 0 & 0 & 0 & 0 & 0 & 1 & 1 \\
\hline 5 & 0 & 0 & 0 & 0 & 0 & 0 & 0 & 0 & 0 & 0 & 0 & 0 & 0 & 0 & 0 & 1 & 1 \\
\hline 6 & 0 & 2 & 0 & 0 & 2 & 0 & 0 & 0 & 0 & 0 & 0 & 0 & 0 & 0 & 0 & 1 & 1 \\
\hline 7 & 0 & 0 & 1 & 1 & 2 & 0 & 2 & 0 & 2 & 2 & 0 & 1 & 2 & 2 & 0 & 3 & 2 \\
\hline 8 & 0 & 0 & 0 & 2 & 2 & 0 & 0 & 0 & 0 & 0 & 2 & 2 & 0 & 0 & 0 & 1 & 1 \\
\hline 9 & 0 & 2 & 2 & 2 & 2 & 0 & 2 & 0 & 0 & 2 & 0 & 2 & 0 & 0 & 0 & 1 & 1 \\
\hline 10 & 0 & 0 & 0 & 0 & 0 & 0 & 0 & 0 & 0 & 0 & 0 & 0 & 0 & 0 & 0 & 1 & 1 \\
\hline 11 & 0 & 0 & 0 & 0 & 2 & 0 & 0 & 0 & 0 & 0 & 0 & 0 & 0 & 0 & 0 & 1 & 1 \\
\hline 12 & 0 & 2 & 0 & 0 & 0 & 0 & 2 & 0 & 0 & 0 & 0 & 0 & 0 & 0 & 0 & 1 & 1 \\
\hline 13 & 2 & 0 & 1 & 1 & 1 & 2 & 1 & 0 & 2 & 1 & 2 & 1 & 2 & 1 & 1 & 3 & 2 \\
\hline 14 & 3 & 3 & 3 & 3 & 3 & 3 & 3 & 3 & 3 & 3 & 3 & 3 & 3 & 3 & 3 & 2 & \\
\hline 15 & 0 & 2 & 0 & 0 & 0 & 0 & 0 & 0 & 0 & 0 & 0 & 0 & 0 & 0 & 0 & 1 & 1 \\
\hline 16 & 0 & 2 & 0 & 0 & 2 & 0 & 0 & 0 & 0 & 0 & 0 & 0 & 0 & 0 & 0 & 1 & 1 \\
\hline 17 & 0 & 0 & 0 & 0 & 2 & 0 & 0 & 0 & 0 & 0 & 0 & 3 & 0 & 0 & 0 & 1 & 1 \\
\hline 18 & 0 & 0 & 0 & 0 & 0 & 0 & 0 & 0 & 0 & 0 & 0 & 0 & 0 & 0 & 0 & 1 & 1 \\
\hline 19 & 0 & 0 & 0 & 2 & 2 & 0 & 2 & 0 & 0 & 0 & 0 & 0 & 0 & 0 & 0 & 1 & 1 \\
\hline 20 & 0 & 0 & 0 & 3 & 2 & 0 & 0 & 0 & 0 & 0 & 3 & 3 & 0 & 0 & 0 & 1 & 1 \\
\hline 21 & 0 & 1 & 0 & 0 & 0 & 0 & 2 & 0 & 0 & 0 & 0 & 0 & 0 & 0 & 0 & 1 & 1 \\
\hline 22 & 0 & 2 & 0 & 0 & 0 & 0 & 0 & 0 & 0 & 0 & 0 & 0 & 0 & 0 & 0 & 1 & 1 \\
\hline 23 & 0 & 2 & 0 & 2 & 2 & 0 & 0 & 0 & 0 & 0 & 3 & 3 & 0 & 0 & 0 & 1 & 1 \\
\hline 24 & 0 & 0 & 0 & 0 & 0 & 0 & 0 & 0 & 0 & 0 & 0 & 0 & 0 & 0 & 0 & 1 & 1 \\
\hline 25 & 0 & 1 & 0 & 0 & 0 & 0 & 2 & 0 & 0 & 0 & 0 & 0 & 0 & 0 & 0 & 1 & 1 \\
\hline 26 & 0 & 0 & 0 & 0 & 0 & 0 & 0 & 0 & 0 & 0 & 0 & 0 & 2 & 0 & 0 & 1 & 1 \\
\hline 27 & 2 & 0 & 1 & 1 & 1 & 0 & 1 & 0 & 2 & 1 & 2 & 1 & 2 & 1 & 2 & 3 & 2 \\
\hline 28 & 0 & 2 & 0 & 0 & 3 & 0 & 0 & 0 & 0 & 0 & 0 & 0 & 0 & 0 & 0 & 1 & 1 \\
\hline 29 & 3 & 3 & 3 & 3 & 3 & 3 & 3 & 3 & 0 & 0 & 0 & 0 & 0 & 0 & 0 & 2 & \\
\hline 30 & 0 & 0 & 2 & 0 & 2 & 0 & 0 & 0 & 0 & 0 & 0 & 0 & 0 & 0 & 0 & 1 & 1 \\
\hline 31 & 0 & 0 & 0 & 2 & 2 & 0 & 0 & 0 & 0 & 0 & 0 & 0 & 0 & 0 & 0 & 1 & 1 \\
\hline 32 & 0 & 0 & 3 & 2 & 2 & 0 & 0 & 0 & 2 & 0 & 0 & 3 & 0 & 0 & 0 & 1 & 1 \\
\hline 33 & 0 & 0 & 0 & 2 & 2 & 2 & 2 & 0 & 0 & 0 & 0 & 0 & 0 & 0 & 0 & 1 & 1 \\
\hline 34 & 3 & 3 & 3 & 3 & 3 & 3 & 3 & 3 & 3 & 3 & 3 & 3 & 3 & 3 & 3 & 2 & \\
\hline 35 & 0 & 2 & 0 & 0 & 0 & 0 & 3 & 0 & 0 & 0 & 0 & 0 & 0 & 0 & 0 & 1 & 1 \\
\hline 35 & 0 & 2 & 0 & 0 & 0 & 0 & 3 & 0 & 0 & 0 & 0 & 0 & 0 & 0 & 0 & 1 & 1 \\
\hline 36 & 0 & 0 & 0 & 2 & 2 & 0 & 0 & 0 & 0 & 0 & 0 & 0 & 0 & 0 & 0 & 1 & 1 \\
\hline 37 & 0 & 1 & 0 & 0 & 0 & 0 & 0 & 0 & 0 & 0 & 0 & 0 & 0 & 0 & 0 & 1 & 1 \\
\hline 38 & 0 & 2 & 0 & 0 & 0 & 0 & 0 & 0 & 0 & 0 & 3 & 0 & 0 & 0 & 0 & 1 & 1 \\
\hline 39 & 0 & 1 & 0 & 0 & 0 & 0 & 2 & 0 & 0 & 0 & 0 & 0 & 0 & 0 & 0 & 1 & 1 \\
\hline 40 & 0 & 0 & 3 & 3 & 3 & 3 & 0 & 0 & 3 & 0 & 3 & 3 & 0 & 0 & 0 & 1 & 1 \\
\hline 41 & 0 & 1 & 0 & 0 & 0 & 0 & 0 & 0 & 0 & 0 & 0 & 0 & 0 & 0 & 0 & 1 & 1 \\
\hline 42 & 0 & 0 & 1 & 1 & 2 & 0 & 2 & 0 & 2 & 2 & 0 & 1 & 2 & 0 & 2 & 3 & 2 \\
\hline 43 & 0 & 0 & 0 & 0 & 2 & 0 & 0 & 0 & 0 & 0 & 0 & 0 & 0 & 0 & 0 & 1 & 1 \\
\hline 44 & 3 & 3 & 3 & 3 & 3 & 3 & 3 & 3 & 3 & 3 & 3 & 3 & 3 & 3 & 3 & 2 & \\
\hline 45 & 0 & 0 & 2 & 2 & 2 & 0 & 0 & 0 & 0 & 0 & 0 & 0 & 0 & 0 & 0 & 1 & 1 \\
\hline 46 & 0 & 0 & 0 & 0 & 2 & 0 & 0 & 0 & 0 & 0 & 0 & 0 & 0 & 0 & 0 & 1 & 1 \\
\hline 47 & 0 & 0 & 0 & 0 & 2 & 0 & 0 & 0 & 0 & 0 & 0 & 0 & 0 & 0 & 0 & 1 & 1 \\
\hline 48 & 0 & 0 & 0 & 0 & 2 & 0 & 0 & 0 & 0 & 0 & 0 & 0 & 0 & 0 & 0 & 1 & 1 \\
\hline 49 & 0 & 0 & 3 & 0 & 2 & 0 & 0 & 0 & 0 & 0 & 0 & 0 & 0 & 0 & 0 & 1 & 1 \\
\hline
\end{tabular}


Table A7.2-continued

\begin{tabular}{|c|c|c|c|c|c|c|c|c|c|c|c|c|c|c|c|c|c|}
\hline$(*)$ & $A$ & B & C & $\mathrm{D}$ & $\mathrm{E}$ & $\mathrm{F}$ & $\mathrm{G}$ & $\mathrm{H}$ & $\mathrm{J}$ & $\mathrm{K}$ & L & M & $N$ & $\mathrm{O}$ & $\mathrm{P}$ & SC1 & $\mathrm{SC} 2$ \\
\hline 50 & 0 & 2 & 0 & 0 & 0 & 0 & 0 & 0 & 0 & 0 & 2 & 0 & 0 & 0 & 0 & 1 & 1 \\
\hline 51 & 2 & 0 & 1 & 1 & 1 & 2 & 1 & 0 & 2 & 1 & 2 & 1 & 2 & 1 & 1 & 3 & 2 \\
\hline 52 & 0 & 0 & 0 & 0 & 0 & 0 & 0 & 0 & 0 & 0 & 0 & 0 & 0 & 0 & 0 & 1 & 1 \\
\hline 53 & 0 & 0 & 0 & 0 & 0 & 0 & 0 & 0 & 0 & 0 & 0 & 2 & 0 & 0 & 0 & 1 & 1 \\
\hline 54 & 2 & 0 & 1 & 1 & 1 & 2 & 1 & 0 & 2 & 1 & 0 & 1 & 2 & 1 & 1 & 3 & 2 \\
\hline 55 & 0 & 0 & 0 & 0 & 0 & 0 & 2 & 0 & 0 & 0 & 0 & 0 & 0 & 0 & 0 & 1 & 1 \\
\hline 56 & 0 & 0 & 2 & 0 & 0 & 0 & 2 & 0 & 0 & 0 & 0 & 0 & 0 & 0 & 0 & 1 & 1 \\
\hline 57 & 0 & 1 & 0 & 0 & 0 & 0 & 2 & 0 & 0 & 0 & 0 & 0 & 0 & 0 & 0 & 1 & 1 \\
\hline 58 & 0 & 0 & 0 & 0 & 0 & 0 & 0 & 0 & 0 & 0 & 0 & 0 & 0 & 0 & 0 & 1 & 1 \\
\hline 59 & 0 & 2 & 0 & 0 & 2 & 0 & 0 & 0 & 0 & 0 & 0 & 2 & 2 & 0 & 0 & 1 & 1 \\
\hline 60 & 3 & 3 & 3 & 3 & 3 & 3 & 3 & 3 & 3 & 3 & 3 & 3 & 3 & 3 & 3 & 2 & \\
\hline 61 & 3 & 3 & 3 & 3 & 3 & 0 & 0 & 0 & 0 & 0 & 0 & 0 & 0 & 0 & 0 & 1 & 1 \\
\hline 62 & 0 & 0 & 0 & 0 & 0 & 0 & 0 & 0 & 0 & 0 & 0 & 0 & 0 & 0 & 0 & 1 & 1 \\
\hline 63 & 3 & 3 & 3 & 3 & 3 & 3 & 3 & 3 & 3 & 3 & 3 & 3 & 3 & 3 & 3 & 2 & \\
\hline 64 & 0 & 0 & 0 & 0 & 0 & 0 & 0 & 0 & 0 & 0 & 0 & 0 & 0 & 0 & 0 & 1 & 1 \\
\hline 65 & 0 & 0 & 0 & 2 & 2 & 0 & 2 & 2 & 0 & 0 & 0 & 0 & 0 & 0 & 0 & 1 & 1 \\
\hline 66 & 0 & 0 & 2 & 2 & 2 & 0 & 0 & 0 & 0 & 0 & 0 & 0 & 0 & 0 & 0 & 1 & 1 \\
\hline 67 & 0 & 1 & 0 & 0 & 0 & 0 & 2 & 0 & 0 & 0 & 0 & 0 & 0 & 0 & 0 & 1 & 1 \\
\hline 68 & 0 & 0 & 1 & 1 & 2 & 0 & 2 & 0 & 2 & 1 & 0 & 1 & 2 & 2 & 2 & 3 & 2 \\
\hline 69 & 0 & 2 & 0 & 0 & 0 & 0 & 0 & 0 & 0 & 0 & 2 & 0 & 0 & 0 & 0 & 1 & 1 \\
\hline 70 & 0 & 0 & 0 & 0 & 0 & 0 & 0 & 0 & 0 & 0 & 3 & 0 & 0 & 0 & 0 & 1 & 1 \\
\hline 71 & 0 & 0 & 0 & 0 & 0 & 0 & 0 & 0 & 0 & 0 & 2 & 0 & 0 & 0 & 0 & 1 & 1 \\
\hline 72 & 0 & 0 & 0 & 0 & 0 & 0 & 3 & 0 & 0 & 0 & 0 & 0 & 0 & 0 & 0 & 1 & 1 \\
\hline 73 & 0 & 0 & 2 & 0 & 2 & 0 & 0 & 0 & 0 & 0 & 0 & 2 & 0 & 0 & 0 & 1 & 1 \\
\hline 74 & 0 & 0 & 1 & 1 & 1 & 2 & 1 & 2 & 2 & 2 & 2 & 1 & 2 & 1 & 2 & 3 & 2 \\
\hline 75 & 2 & 0 & 1 & 1 & 1 & 2 & 1 & 0 & 2 & 1 & 2 & 1 & 2 & 1 & 1 & 3 & 2 \\
\hline 76 & 0 & 0 & 2 & 3 & 2 & 0 & 0 & 0 & 2 & 0 & 0 & 0 & 0 & 0 & 0 & 1 & 1 \\
\hline 77 & 0 & 0 & 3 & 2 & 3 & 0 & 0 & 0 & 0 & 0 & 0 & 3 & 0 & 0 & 0 & 1 & 1 \\
\hline 78 & 2 & 0 & 1 & 1 & 1 & 0 & 2 & 2 & 0 & 2 & 0 & 1 & 2 & 2 & 2 & 3 & 2 \\
\hline 79 & 0 & 0 & 0 & 0 & 0 & 0 & 0 & 0 & 0 & 0 & 0 & 0 & 0 & 0 & 0 & 1 & 1 \\
\hline 80 & 0 & 0 & 0 & 0 & 0 & 0 & 0 & 0 & 0 & 0 & 0 & 0 & 0 & 0 & 0 & 1 & 1 \\
\hline 81 & 0 & 0 & 0 & 0 & 3 & 0 & 0 & 0 & 0 & 0 & 0 & 0 & 0 & 0 & 0 & 1 & 1 \\
\hline 82 & 0 & 1 & 0 & 0 & 0 & 0 & 0 & 0 & 0 & 0 & 0 & 0 & 0 & 0 & 0 & 1 & 1 \\
\hline 83 & 0 & 0 & 0 & 0 & 3 & 0 & 0 & 0 & 0 & 0 & 0 & 0 & 0 & 0 & 0 & 1 & 1 \\
\hline 84 & 0 & 0 & 0 & 0 & 0 & 0 & 0 & 0 & 0 & 0 & 0 & 0 & 0 & 0 & 0 & 1 & 1 \\
\hline 85 & 0 & 0 & 0 & 0 & 2 & 0 & 0 & 0 & 0 & 0 & 2 & 0 & 0 & 0 & 0 & 1 & 1 \\
\hline 86 & 0 & 3 & 0 & 0 & 0 & 0 & 0 & 0 & 0 & 0 & 0 & 0 & 0 & 0 & 0 & 1 & 1 \\
\hline 87 & 2 & 0 & 1 & 1 & 2 & 2 & 1 & 0 & 2 & 1 & 2 & 1 & 2 & 1 & 2 & 3 & 2 \\
\hline 88 & 0 & 2 & 0 & 0 & 0 & 0 & 0 & 0 & 0 & 0 & 0 & 0 & 0 & 0 & 0 & 1 & 1 \\
\hline 89 & 0 & 0 & 0 & 0 & 0 & 0 & 2 & 0 & 0 & 0 & 0 & 0 & 3 & 0 & 0 & 1 & 1 \\
\hline 90 & 0 & 0 & 0 & 0 & 0 & 0 & 0 & 0 & 0 & 0 & 0 & 0 & 0 & 0 & 0 & 1 & 1 \\
\hline 91 & 0 & 0 & 0 & 0 & 2 & 0 & 0 & 0 & 0 & 0 & 0 & 0 & 0 & 0 & 0 & 1 & 1 \\
\hline 92 & 0 & 0 & 0 & 0 & 0 & 0 & 0 & 0 & 0 & 0 & 0 & 0 & 0 & 0 & 0 & 1 & 1 \\
\hline 93 & 0 & 3 & 3 & 3 & 3 & 0 & 0 & 0 & 0 & 0 & 0 & 3 & 0 & 0 & 0 & 1 & 1 \\
\hline 94 & 0 & 0 & 0 & 0 & 0 & 0 & 0 & 0 & 0 & 0 & 0 & 0 & 0 & 0 & 0 & 1 & 1 \\
\hline 95 & 0 & 2 & 0 & 0 & 0 & 0 & 0 & 0 & 0 & 0 & 0 & 0 & 0 & 0 & 0 & 1 & 1 \\
\hline 96 & 0 & 2 & 0 & 0 & 0 & 0 & 0 & 0 & 0 & 0 & 0 & 0 & 0 & 0 & 0 & 1 & 1 \\
\hline 97 & 0 & 1 & 0 & 0 & 0 & 0 & 2 & 0 & 0 & 0 & 0 & 0 & 0 & 0 & 0 & 1 & 1 \\
\hline 98 & 0 & 0 & 0 & 0 & 2 & 0 & 0 & 0 & 0 & 0 & 0 & 3 & 0 & 0 & 0 & 1 & 1 \\
\hline 99 & 0 & 2 & 0 & 0 & 0 & 0 & 3 & 0 & 0 & 0 & 0 & 0 & 0 & 0 & 0 & 1 & 1 \\
\hline 100 & 0 & 0 & 0 & 0 & 2 & 0 & 0 & 0 & 0 & 0 & 0 & 0 & 2 & 0 & 0 & 1 & 1 \\
\hline
\end{tabular}


Table A7.2-continued

\begin{tabular}{|c|c|c|c|c|c|c|c|c|c|c|c|c|c|c|c|c|c|}
\hline$(*)$ & $\mathrm{A}$ & B & C & $\mathrm{D}$ & $\mathrm{E}$ & $\mathrm{F}$ & $\mathrm{G}$ & $\mathrm{H}$ & $\mathbf{J}$ & $\mathrm{K}$ & $\mathrm{L}$ & $\mathrm{M}$ & $N$ & $\mathrm{O}$ & $P$ & $\mathrm{SC} 1$ & $\mathrm{SC} 2$ \\
\hline 101 & 2 & 0 & 1 & 1 & 2 & 2 & 1 & 0 & 2 & 1 & 2 & 1 & 2 & 1 & 2 & 3 & 2 \\
\hline 102 & 2 & 0 & 1 & 1 & 2 & 0 & 2 & 0 & 2 & 2 & 2 & 1 & 2 & 2 & 2 & 3 & 2 \\
\hline 103 & 0 & 2 & 0 & 0 & 0 & 0 & 0 & 0 & 0 & 0 & 0 & 0 & 0 & 0 & 0 & 1 & 1 \\
\hline 104 & 0 & 0 & 2 & 0 & 0 & 0 & 2 & 0 & 0 & 0 & 0 & 0 & 0 & 0 & 0 & 1 & 1 \\
\hline 105 & 0 & 0 & 0 & 0 & 0 & 0 & 0 & 0 & 0 & 0 & 2 & 0 & 0 & 0 & 0 & 1 & 1 \\
\hline 106 & 0 & 0 & 1 & 1 & 2 & 0 & 2 & 0 & 2 & 1 & 0 & 1 & 2 & 2 & 2 & 3 & 2 \\
\hline 107 & 0 & 0 & 0 & 0 & 2 & 0 & 0 & 0 & 3 & 0 & 0 & 0 & 0 & 0 & 0 & 1 & 1 \\
\hline 108 & 0 & 0 & 0 & 0 & 2 & 0 & 0 & 0 & 0 & 0 & 2 & 0 & 0 & 0 & 0 & 1 & 1 \\
\hline 109 & 0 & 0 & 0 & 0 & 0 & 0 & 0 & 0 & 0 & 0 & 0 & 0 & 0 & 0 & 0 & 1 & 1 \\
\hline 110 & 3 & 3 & 3 & 3 & 3 & 3 & 3 & 3 & 3 & 3 & 3 & 3 & 3 & 3 & 3 & 2 & \\
\hline 111 & 0 & 0 & 2 & 2 & 2 & 0 & 0 & 0 & 2 & 2 & 0 & 2 & 0 & 0 & 0 & 1 & 1 \\
\hline 112 & 0 & 0 & 0 & 0 & 2 & 0 & 0 & 0 & 0 & 0 & 0 & 0 & 0 & 0 & 0 & 1 & 1 \\
\hline 113 & 0 & 0 & 3 & 2 & 2 & 0 & 2 & 0 & 0 & 0 & 0 & 3 & 0 & 0 & 0 & 1 & 1 \\
\hline 114 & 0 & 1 & 0 & 0 & 0 & 0 & 2 & 0 & 0 & 0 & 0 & 0 & 0 & 0 & 0 & 1 & 1 \\
\hline 115 & 2 & 0 & 1 & 1 & 1 & 0 & 1 & 0 & 0 & 1 & 0 & 1 & 2 & 1 & 2 & 3 & 2 \\
\hline 116 & 0 & 0 & 0 & 0 & 0 & 0 & 0 & 0 & 0 & 0 & 0 & 0 & 0 & 0 & 0 & 1 & 1 \\
\hline 117 & 0 & 3 & 0 & 0 & 0 & 0 & 3 & 0 & 0 & 0 & 0 & 0 & 0 & 0 & 0 & 1 & 1 \\
\hline 118 & 3 & 3 & 3 & 3 & 3 & 3 & 3 & 0 & 0 & 0 & 0 & 0 & 0 & 0 & 0 & 1 & 1 \\
\hline 119 & 0 & 0 & 0 & 2 & 2 & 0 & 0 & 0 & 0 & 2 & 0 & 0 & 0 & 0 & 0 & 1 & 1 \\
\hline 120 & 0 & 0 & 0 & 0 & 0 & 0 & 0 & 0 & 0 & 0 & 0 & 0 & 0 & 3 & 0 & 1 & 1 \\
\hline 121 & 3 & 3 & 3 & 3 & 3 & 3 & 3 & 3 & 3 & 3 & 3 & 3 & 3 & 3 & 3 & 2 & \\
\hline 122 & 0 & 2 & 3 & 0 & 3 & 0 & 3 & 0 & 0 & 0 & 0 & 0 & 0 & 0 & 0 & 1 & 1 \\
\hline 123 & 3 & 3 & 3 & 3 & 3 & 3 & 3 & 0 & 0 & 0 & 0 & 0 & 0 & 0 & 0 & 1 & 1 \\
\hline 124 & 0 & 0 & 2 & 0 & 2 & 0 & 0 & 0 & 0 & 0 & 0 & 3 & 0 & 0 & 0 & 1 & 1 \\
\hline 125 & 0 & 0 & 0 & 0 & 0 & 0 & 0 & 0 & 0 & 0 & 3 & 0 & 0 & 0 & 0 & 1 & 1 \\
\hline 126 & 0 & 0 & 0 & 0 & 0 & 0 & 0 & 0 & 0 & 0 & 0 & 0 & 0 & 0 & 0 & 1 & 1 \\
\hline 127 & 0 & 0 & 0 & 3 & 2 & 0 & 0 & 0 & 3 & 0 & 3 & 3 & 0 & 0 & 0 & 1 & 1 \\
\hline 128 & 3 & 3 & 3 & 3 & 3 & 3 & 3 & 3 & 3 & 3 & 3 & 3 & 3 & 3 & 3 & 2 & \\
\hline 129 & 3 & 0 & 0 & 0 & 2 & 0 & 0 & 0 & 0 & 0 & 0 & 0 & 0 & 0 & 0 & 1 & 1 \\
\hline 130 & 0 & 0 & 1 & 1 & 2 & 0 & 2 & 0 & 0 & 2 & 0 & 1 & 2 & 2 & 2 & 3 & 2 \\
\hline 131 & 0 & 0 & 0 & 0 & 2 & 0 & 0 & 0 & 0 & 0 & 0 & 0 & 0 & 0 & 0 & 1 & 1 \\
\hline 132 & 3 & 3 & 3 & 3 & 3 & 3 & 3 & 3 & 3 & 3 & 3 & 3 & 3 & 3 & 3 & 2 & \\
\hline 133 & 0 & 0 & 0 & 0 & 2 & 0 & 0 & 0 & 0 & 0 & 0 & 0 & 0 & 0 & 0 & 1 & 1 \\
\hline 134 & 0 & 2 & 0 & 0 & 0 & 3 & 0 & 0 & 0 & 0 & 0 & 0 & 0 & 0 & 0 & 1 & 1 \\
\hline 135 & 3 & 3 & 3 & 3 & 3 & 3 & 3 & 3 & 3 & 3 & 3 & 3 & 3 & 3 & 3 & 2 & \\
\hline 136 & 0 & 0 & 2 & 0 & 2 & 0 & 0 & 0 & 0 & 0 & 2 & 2 & 0 & 0 & 0 & 1 & 1 \\
\hline 137 & 0 & 1 & 0 & 0 & 0 & 0 & 0 & 0 & 0 & 0 & 0 & 0 & 0 & 0 & 0 & 1 & 1 \\
\hline 138 & 0 & 0 & 0 & 0 & 2 & 0 & 0 & 0 & 0 & 0 & 3 & 0 & 0 & 0 & 0 & 1 & 1 \\
\hline 139 & 0 & 0 & 0 & 0 & 0 & 0 & 0 & 0 & 0 & 0 & 0 & 0 & 0 & 0 & 0 & 1 & 1 \\
\hline 140 & 0 & 0 & 0 & 0 & 0 & 0 & 0 & 0 & 0 & 0 & 0 & 0 & 0 & 0 & 0 & 1 & 1 \\
\hline 141 & 0 & 0 & 0 & 0 & 0 & 0 & 0 & 0 & 0 & 0 & 0 & 0 & 0 & 0 & 0 & 1 & 1 \\
\hline 142 & 2 & 0 & 1 & 1 & 1 & 2 & 1 & 0 & 2 & 1 & 0 & 1 & 2 & 1 & 2 & 3 & 2 \\
\hline 143 & 0 & 2 & 0 & 0 & 0 & 0 & 0 & 0 & 0 & 0 & 0 & 0 & 0 & 0 & 0 & 1 & 1 \\
\hline 144 & 0 & 1 & 0 & 0 & 0 & 0 & 2 & 0 & 0 & 0 & 0 & 0 & 0 & 0 & 0 & 1 & 1 \\
\hline 145 & 0 & 1 & 0 & 0 & 0 & 0 & 2 & 0 & 0 & 0 & 0 & 0 & 0 & 0 & 0 & 1 & 1 \\
\hline 146 & 0 & 0 & 0 & 0 & 0 & 0 & 0 & 0 & 0 & 0 & 0 & 0 & 0 & 0 & 0 & 1 & 1 \\
\hline 147 & 2 & 0 & 1 & 1 & 1 & 2 & 1 & 0 & 2 & 1 & 2 & 1 & 2 & 2 & 1 & 3 & 2 \\
\hline 148 & 0 & 0 & 0 & 0 & 0 & 0 & 0 & 0 & 0 & 0 & 0 & 0 & 0 & 0 & 0 & 1 & 1 \\
\hline 149 & 1 & 0 & 1 & 1 & 1 & 2 & 1 & 1 & 2 & 1 & 2 & 1 & 1 & 1 & 1 & 3 & 2 \\
\hline 150 & 0 & 0 & 2 & 2 & 2 & 0 & 0 & 0 & 0 & 0 & 0 & 0 & 0 & 0 & 0 & 1 & 1 \\
\hline 151 & 3 & 3 & 3 & 3 & 3 & 3 & 3 & 3 & 3 & 3 & 3 & 3 & 3 & 3 & 3 & 2 & \\
\hline
\end{tabular}


Table A7.2-continued

\begin{tabular}{llllllllllllllllll}
\hline$*)$ & A & B & C & D & E & F & G & H & J & K & L & M & N & O & P & SC1 & SC2 \\
\hline 152 & 0 & 2 & 0 & 0 & 2 & 0 & 0 & 0 & 0 & 0 & 0 & 0 & 0 & 0 & 0 & 1 & 1 \\
153 & 0 & 1 & 0 & 0 & 0 & 0 & 0 & 0 & 0 & 0 & 0 & 0 & 0 & 0 & 0 & 1 & 1 \\
154 & 0 & 0 & 0 & 0 & 0 & 0 & 0 & 0 & 0 & 0 & 0 & 0 & 0 & 0 & 0 & 1 & 1 \\
155 & 0 & 2 & 0 & 0 & 0 & 0 & 0 & 0 & 0 & 0 & 0 & 0 & 0 & 0 & 0 & 1 & 1 \\
156 & 0 & 0 & 2 & 2 & 2 & 0 & 0 & 0 & 0 & 0 & 0 & 0 & 0 & 0 & 0 & 1 & 1 \\
157 & 0 & 0 & 0 & 0 & 0 & 0 & 0 & 0 & 0 & 0 & 0 & 0 & 0 & 0 & 0 & 1 & 1 \\
158 & 3 & 3 & 3 & 0 & 0 & 0 & 0 & 0 & 0 & 0 & 0 & 0 & 0 & 0 & 0 & 1 & 1 \\
\hline
\end{tabular}

(0): In favour, (1): Against, (2): Abstaining, (3): Absent.

(*) The States 1 to 158 are precisely those given in Table A7.1.

\section{A7.3. Votes on Resolution 39/99: United Nations Relief and Works for Palestine Refugees in the Near East}

The parameters are specified as follows:

A: Assistance to Palestine refugees. D: Offers by Member States of grants and scholarships for higher education including vocational training, for the Palestine refugees. E: Palestine refugees in the Gaza Strip. F: Resumption of the ration distribution to Palestine refugees. G: Population and refugees displaced since 1967. H: Revenues derived from Palestine refugee properties. I: Protection of Palestine refugees. J: Palestine refugees in the West Bank. K: University of Jerusalem "Al-Quds" for Palestine refugees.

For the data matrix see UNO (1985) or Wakabayashi (1986). Consider the states from 1 to 158 as in Table A7.1 and the 9 motions above specified. The solution classes are specified as follows:

Solution classes considering all states:

Class 1: $5,34,44,60,71,119,120,128,132,135,151$. Class 2: 74,149 . Class 3 : all the others.

Solution classes without considering states which were absent at least 5 times:

Class 1: 74,149 . Class 2: all the others.

\section{Appendix A8. Classification of companies}

Reference: Späth (1977). The complete data set can be found in Späth (1977). The solutions classes are specified as follows:

Class 1: 10 and 107. Class 2: all the others.

\section{Acknowledgement}

The authors would like to thank O. Opitz (Augsburg) for introducing them to qualitative data analysis and suggesting the research project on which this paper reports.

\section{References}

F. Barahona, M. Grötschel, M. Jünger and G. Reinelt, "An application of combinatorial optimization to statistical physics and circuit layout design," Operations Research 36 (1988) 493-513. 
J.P. Barthélemy and B. Monjardet, "The median procedure in cluster analysis and social choice theory," Mathematical Social Sciences 1 (1981) 235-267.

J.A. Bondy and U.S.R. Murty, Graph Theory with Applications (Macmillan, London, 1976).

S. Chah, "Classification of heterogeneous data: micro computers," Paper presented at the III International Symposium on Data Analysis (Brussels, 1985).

H.P. Crowder and M.W. Padberg, "Solving large scale travelling salesman problems to optimality," Management Science 26 (1980) 495-509.

M. Grötschel, L. Lovász and A. Schrijver, "The ellipsoid method and its consequences in combinatorial optimization", Combinatorica 1 (1981) 169-197.

M. Grötschel, M. Jünger and G. Reinelt, "A cutting plane algorithm for the linear ordering problem", Operations Research 32 (1984) 1195-1220.

M. Grötschel and O. Holland, "Solving matching problems with linear programming," Mathematical Programming 33 (1985) 243-259.

M. Grötschel and Y. Wakabayashi, "Facets of the clique partitioning polytope", Report No. 6, Schwerpunktprogramm der Deutschen Forschungsgemeinschaft Universität Augsburg (Augsburg, West Germany, 1987), to appear in Mathematical Programming.

J.A. Hartigan, Clustering Algorithms (Wiley, New York, 1975).

J.F. Marcotorchino, Agrégation des Similarités en Classification Automatique, These de Doctorat d'état (Université Paris vi, 1981).

J.F. Marcotorchino and P. Michaud, "Optimisation en analyse des données relationnelles," in: E. Diday, et al. (eds.), Data Analysis and Informatics (North-Holland, Amsterdam, 1980) pp. 655-670.

J.F. Marcotorchino and P. Michaud, "Optimization in exploratory data analysis," Proceedings of 5th International Symposium on Operations Research 1981 (Physica Verlag, Köln, 1981a).

J.F. Marcotorchino and P. Michaud, "Heuristic approach to the similarity aggregation problem," Methods of Operations Research 43 (1981b) 395-404.

O. Opitz and M. Schader, "Analyse qualitativer Daten: Einführung und Übersicht," Operations Research Spektrum 6 (1984) 67-83.

M. Padberg and G. Rinaldi, "Optimization of a 532-city symmetric traveling salesman problem by branch and cut," Operations Research Letters 6 (1987) 1-7.

G. Reinelt, The Linear Ordering Problem: Algorithms and Applications (Research and Exposition in Mathematics 8) (Helderman Verlag, Berlin, 1985).

M. Schader and U. Tüshaus, "Ein Subgradientenverfahren zur Klassifikation qualitativer Daten," Operations Research Spektrum 7 (1985) 1-5.

A. Schrijver, Theory of Linear and Integer Programming (Wiley, Chichester, UK, 1986).

H. Späth, "Partitionierende Cluster-Analyse für große Objektmengen mit binären Merkmalen am Beispiel von Firmen und deren Berufsgruppenbedarf," in: H. Späth, (ed.), Fallstudien Cluster Analyse (Oldenburg, München, 1977) pp. 63-80.

U. Tüshaus, "Aggregation binärer Relationen in der qualitativen Datenanalyse," in: Mathematical Systems in Economics Vol. 82 (Hain, Königstein, 1983).

UNO, "Resolutions and Decisions adopted by the General Assembly during the first part of its thirty-ninth Session" (from Sept. 18 to Dec. 18, 1984), (1985) 412-419.

G. Vescia, (a) "Descriptive classification of cetacea: whales, porpoises and dolphins," (b) "Automatic classification of cetaceans by similarity aggregation," in: J.F. Marcotorchino, J.M. Proth and J. Janssen, (eds.), Data Analysis in Real Life Environment: Ins and Outs of Solving Problems (Elsevier Science Publishers B.V. (North-Holland, Amsterdam, 1985) pp. 7-24.

Y. Wakabayashi, Aggregation of Binary Relations: Algorithmic and Polyhedral Investigations, Ph.D. Thesis (Universität Augsburg, West Germany, 1986). 